\title{
KINEMATICS IN KAPTEYN'S SELECTED AREA 76: ORBITAL MOTIONS WITHIN THE HIGHLY SUBSTRUCTURED ANTICENTER STREAM
}

\author{
Jeffrey L. Carlin ${ }^{1,5}$, Dana I. Casetti-Dinescu ${ }^{2,3}$, Carl J. Grillmair ${ }^{4}$, Steven R. Majewski $^{1}$, And Terrence M. Girard ${ }^{2}$ \\ ${ }^{1}$ Department of Astronomy, University of Virginia, P.O. Box 400325, Charlottesville, VA 22904-4325, USA; jc4qn@ mail.astro.virginia.edu, srm4n@ virginia.edu \\ ${ }^{2}$ Astronomy Department, Yale University, P.O. Box 208101, New Haven, CT 06520-8101, USA; dana@ astro.yale.edu, girard@ astro.yale.edu \\ ${ }^{3}$ Astronomical Institute of the Romanian Academy, Str. Cutitul de Argint 5, RO-75212, Bucharest 28, Romania \\ ${ }^{4}$ Spitzer Science Center, 1200 E. California Blvd., Pasadena, CA 91125, USA; carl@ipac.caltech.edu \\ Received 2010 June 30; accepted 2010 October 10; published 2010 December 6
}

\begin{abstract}
We have measured the mean three-dimensional kinematics of stars in Kapteyn's Selected Area (SA) $76(l=$ $209^{\circ} 3, b=26^{\circ}$.4) that were selected to be Anticenter Stream (ACS) members on the basis of their radial velocities (RVs), proper motions (PMs), and location in the color-magnitude diagram. From a total of 31 stars ascertained to be ACS members primarily from its main-sequence turnoff, a mean ACS RV (derived from spectra obtained with the Hydra multi-object spectrograph on the WIYN $3.5 \mathrm{~m}$ telescope) of $V_{\text {helio }}=97.0 \pm 2.8 \mathrm{~km} \mathrm{~s}^{-1}$ was determined, with an intrinsic velocity dispersion $\sigma_{o}=12.8 \pm 2.1 \mathrm{~km} \mathrm{~s}^{-1}$. The mean absolute PMs of these


to the ACS of $10 \pm 3 \mathrm{kpc}$, these measured kinematical quantities produce an orbit that deviates by $\sim 30^{\circ}$ from the well-defined swath of stellar overdensity constituting the ACS in the western portion of the Sloan Digital Sky Survey footprint. We explore possible explanations for this and suggest that our data in SA 76 are measuring the motion of a kinematically cold sub-stream among the ACS debris that was likely a fragment of the same infalling structure that created the larger ACS system. The ACS is clearly separated spatially from the majority of claimed Monoceros ring detections in this region of the sky; however, with the data in hand, we are unable to either confirm or rule out an association between the ACS and the poorly understood Monoceros structure.
\end{abstract}

Key words: astrometry - galaxies: dwarf - Galaxy: kinematics and dynamics - Galaxy: stellar content - Galaxy: structure - Local Group - stars: abundances - stars: kinematics and dynamics

Online-only material: color figures

\section{INTRODUCTION}

\subsection{The Anticenter Stream}

One of the many stellar overdensities detected in Sloan Digital Sky Survey (SDSS) data is the so-called Anticenter Stream (ACS) unveiled by Grillmair (2006, hereafter G06) using matched-filter star counts. The ACS feature is seen as a welldefined swath of stellar excess near the Galactic anticenter at moderately low latitudes $18^{\circ} \lesssim b \lesssim 35^{\circ}$ (at roughly constant right ascension, $\alpha_{2000} \approx 125^{\circ}$ ), spanning the full $\sim 65^{\circ}$ declination coverage of the SDSS database in this region. G06 discovered distinct, well-separated narrow "tributaries" or sub-streams within the broad ACS stream; these tributaries are thought to be dynamically distinct components among the remnants of a tidally disrupted dwarf galaxy. Grillmair et al. (2008, hereafter GCM08) measured radial velocities (RVs) in two fields along the ACS and identified the RV signature of the stream. The relatively large measured velocity dispersion for the northernmost of the two fields (ACS-B; $\sigma_{V} \sim 15 \mathrm{~km} \mathrm{~s}^{-1}$ ) is consistent with the dispersion for a tidal remnant of a disrupted dwarf galaxy, but may also result from the sampling

\footnotetext{
5 Visiting Astronomer, Kitt Peak National Observatory, National Optical Astronomy Observatory, which is operated by the Association of Universities for Research in Astronomy (AURA) under cooperative agreement with the National Science Foundation.
}

of more than one of the apparent cold sub-streams within the broader ACS. For the second field, ACS-C, about $23^{\circ}$ south of ACS-B, GCM08 found a dispersion of only $\sim 5 \mathrm{~km} \mathrm{~s}^{-1}$, suggesting that their data in this field sample only one of the kinematically cold "tributaries" making up the larger system. The mean velocities in each field, combined with SDSS/ USNO-B proper motions (PMs) and the locations of stream overdensities, were used by GCM08 to fit an orbit and show that the ACS corresponds to debris lost from an object on a low-inclination, nearly circular, prograde orbit. This GCM08 orbit succeeds in reproducing not only the broad swath cut by the ACS stream in the SDSS footprint, but also suggests that the "Eastern Banded Structure" (EBS) pointed out by G06 at $(\alpha, \delta)_{2000} \sim\left(134^{\circ}, 3^{\circ} .4\right)$ is associated with the ACS as debris from a subsequent (or prior) orbital wrap of the same system.

\subsection{Previously Studied Stellar Overdensities in the Same Region: Monoceros/GASS}

The stellar overdensity in this region of the SDSS database was identified by Belokurov et al. (2006) as being associated with the Monoceros (Mon) Ring, a set of features first reported by Newberg et al. (2002), Yanny et al. (2003), Rocha-Pinto et al. (2003), and Ibata et al. (2003) that seem to form a lowlatitude ring-like structure over a large area near the Galactic 
anticenter. ${ }^{6}$ However, once the smoothly varying background has been removed (G06) the ACS appears to be a separate narrow stream structure, rather than a part of the much larger Monoceros feature. Nevertheless, because many detected overdensities in this region of sky have been attributed to Monoceros, we will compare our ACS findings to existing Monoceros data.

Based on its narrow radial extent and low velocity dispersion, Crane et al. (2003) argued that Mon was the remnant of a tidally disrupted dwarf galaxy. Ibata et al. (2003) followed up with photometric detections spanning $\sim 100^{\circ}$ and suggested that the apparent ring was a result of flaring or warping of the Galactic disk. Evidence of a rather thin radial extent based on main-sequence width (Yanny et al. 2003; Ibata et al. 2003) as well as apparent separation from the edge of the Galactic disk (Rocha-Pinto et al. 2003) favor an accretion origin for the ring. These facts were taken by Crane et al. (2003) along with their measured RV trend with longitude to be consistent with a dynamically young tidal stream on a clearly non-circular orbit (though with low eccentricity). Low-latitude, torus-like features are produced by accretion of satellites on nearly circular orbits roughly coplanar with the Galactic disk in $\Lambda \mathrm{CDM}$ simulations (Abadi et al. 2003; Bullock \& Johnston 2005) and are also visible in the extragalactic systems imaged by Martínez-Delgado et al. (2010). Crane et al. (2003) also noted that a few Galactic globular clusters may be associated with the Monoceros ring in both position and $\mathrm{RV}$ - a point that was further developed by Frinchaboy et al. (2004) to include 11 old open clusters that are also near the Monoceros plane. Conn et al. (2005, 2007, 2008) have continued to map the extent and stellar populations of the ring and have detected overdensities both above and below the Galactic plane, ruling out a warp or flare of the disk as the origin of the feature. However, recent simulations (Kazantzidis et al. 2008; Younger et al. 2008) have shown that interactions of massive subhalos with the Galactic disk can form ring-like features that result from excitation of thin/thick disk stars. On the other hand, recent high-resolution abundance analysis by Chou et al. (2010) has shown that Monoceros stars have chemical abundance patterns similar to those of Milky Way (MW) dwarf spheroidals (dSphs) and unlike stars from the outer MW disk (M.-Y. Chou et al. 2011, in preparation), bolstering the case for a tidal origin for Mon. Further detailed chemodynamical studies of stream stars are clearly needed to assess the origin of this feature and its possible association with the ACS.

Monoceros has been modeled as debris from a tidally disrupting dwarf galaxy by both Peñarrubia et al. (2005) and Martin et al. (2004), both of whose models were constrained so that the progenitor reproduces the putative Canis Major dwarf galaxy (which was discovered by Martin et al. 2004; but cf. RochaPinto et al. 2006; Momany et al. 2006; Moitinho et al. 2006; López-Corredoira et al. 2007 for alternative explanations of this

\footnotetext{
6 This low-latitude structure and other seemingly related features have been christened variously the "Monoceros Stream" (Yanny et al. 2003), the "One Ring" (Ibata et al. 2003), and Galactic Anticenter Stellar Structure (GASS; Rocha-Pinto et al. 2003). It is not clear that the distinct, narrow stream seen in background-subtracted, filtered star-count maps of the SDSS database (Grillmair 2006) is physically associated with the much larger purported association of overdensities comprising Monoceros/GASS. For convenience, we follow the naming convention from G06 and refer to the narrow stream structure uncovered by these authors in the western portion of the SDSS footprint as the "Anticenter Stream," or ACS. To avoid conflating possibly distinct structures, all other overdensities that have been associated with the Monoceros feature in past works will be referred to in this paper as part of Monoceros (Mon). We acknowledge that some or all of these structures may be associated, and if that is found to be the case, the names might thus be interchangeable.
}

apparent stellar overdensity). The Peñarrubia et al. (2005) models incorporated all detections of suspected Monoceros debris known at the time and reproduced the known structure with a disrupting dwarf galaxy on a low-inclination, nearly circular prograde orbit. Subsequent mapping of the extent and stellar populations of the Ring have yet to be incorporated into the models, and to date only a few kinematical constraints have been derived (e.g., Crane et al. 2003; Yanny et al. 2003). One of the only PM results for Monoceros debris, measured by Casetti-Dinescu et al. (2008) in SA 71, produced an orbit in agreement with the Peñarrubia et al. (2005) determination. This SA 71 study illustrated the difficulty of studying low-latitude features-large numbers of accurate PMs supplemented by RVs were necessary to distinguish the kinematical signature of Mon from the overlapping kinematics of Galactic populations.

\subsection{Goals of This Paper}

Here, we present a follow-up to the GCM08 study, supplementing the RVs from that previous work with substantially more measurements from WIYN+Hydra spectroscopy as well as accurate PMs in one of the fields studied there. This field, ACS-C, was originally selected because it coincides with SA 76, one region from the deep PM study in Kapteyn's Selected Areas (SAs) by Majewski (1992) and Casetti-Dinescu et al. (2006, hereafter CD06). From the newly obtained spectra, additional likely RV members of the ACS are identified and used to derive the mean absolute PM (and thus three-dimensional kinematics) of stream stars along this line of sight. This represents one of the most precise absolute PMs yet derived for a MW stellar tidal stream.

The organization of this paper is as follows. Section 2 gives an overview of the PM and spectroscopic data for SA 76 and their handling, including details of both the astrometric and spectroscopic data reduction. The selection of ACS members is detailed in Section 3. Initially, candidates were selected based on broad RV criteria. Additional culling of the sample was performed based on reduced PMs. In Section 4, we present metallicities for stars selected to be ACS members, comparing our results to the expected $[\mathrm{Fe} / \mathrm{H}]$ distribution of foreground stellar populations selected from the Besançon galaxy model. Section 5 discusses the measured kinematics based on our final sample of ACS debris candidates. The mean RV and absolute PMs yield a low-inclination, nearly circular orbit for ACS debris in SA 76. The mean three-dimensional space motion we find for SA 76 ACS candidates is oriented at a $\sim 30^{\circ}$ angle to the prominently visible ACS. Some possible explanations for this are discussed in Section 6-we believe that the motions we have measured represent a kinematically distinct substream from within the more extensive ACS system. Finally, we compare our results to known characteristics of the Monoceros ring in Section 7. From the available data, the ACS appears to be unrelated to Monoceros, but this remains unclear.

\section{THE DATA}

\subsection{Photometry and Proper Motions}

Photometry from SDSS Data Release 5 (DR5; AdelmanMcCarthy et al. 2007) was used for most analysis in this work (DR7 was used for the star-count maps). Throughout this contribution, quoted magnitudes are measured SDSS magnitudes (i.e., not dereddened or extinction corrected); the mean reddening along this line of sight from the dust maps of Schlegel et al. (1998) is $E(B-V)=0.04$. The main sequence of the ACS is 


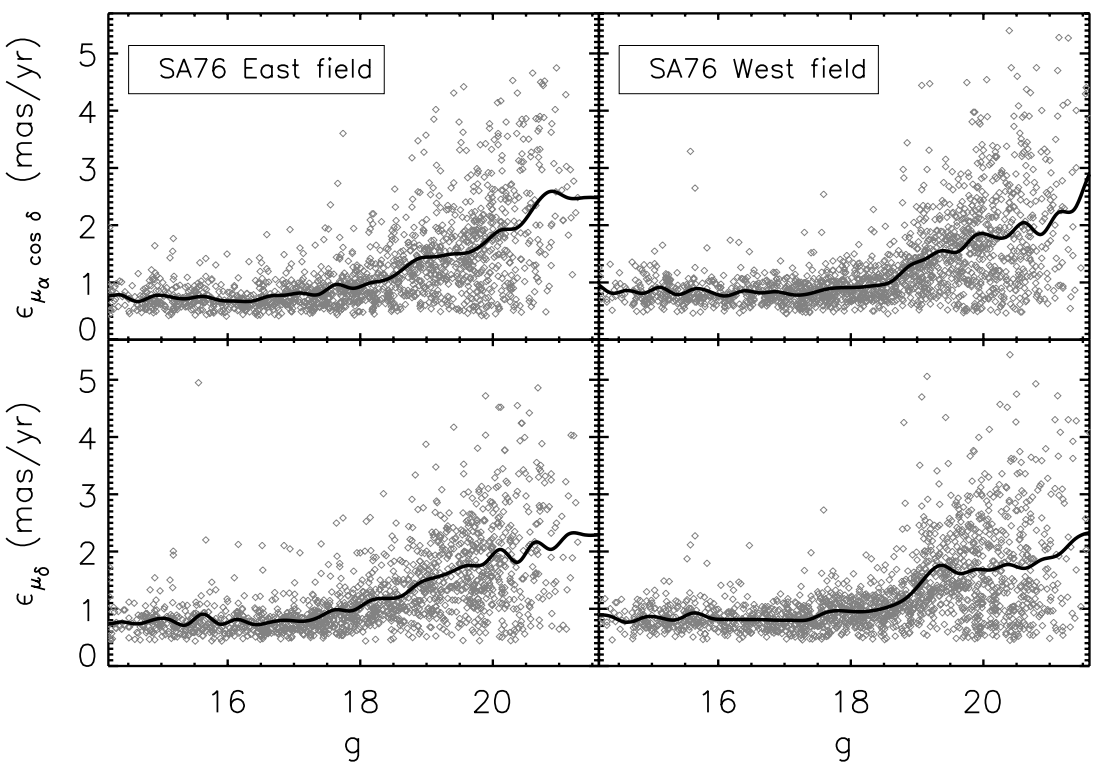

Figure 1. Proper-motion errors as a function of $g$ magnitude for the east and west fields. The solid lines show a moving median in bins of 0.25 mag width. Most well-measured stars have proper-motion uncertainties of $1-2$ mas $\mathrm{yr}^{-1}$ in each direction.

readily distinguished at $g \gtrsim 18.5$ over the large area in which G06 mapped the stream. GCM08 used filtered, backgroundsubtracted number counts (see Grillmair 2009 for details of the matched-filter technique) to identify regions of highest stream density from which to select spectroscopic targets along the ACS. Analysis in GCM08 focused on two widely spaced fields within the ACS: ACS-B at $(\alpha, \delta)_{2000}=\left(124^{\circ}, 37.5\right)$ and ACS-C at $(\alpha, \delta)_{2000}=\left(125^{\circ}, 14.7\right)$. The first of these, ACS-B, was selected because of its relatively high matched-filter stellar density, while the latter field, ACS-C at $(l, b)=\left(209^{\circ} .3,26^{\circ} .4\right)$, was chosen because it overlaps SA 76, one of the regions studied in the deep PM survey first described in (Majewski 1992; see also CD06). We note that SA 76 is offset slightly east of the highest-density portion of the stream, but is still in a region of elevated stellar density.

SA 76 is one field of $\sim 50$ from the Mt. Wilson 60 inch telescope-based part of the PM survey described by CD06; details concerning the data reduction process and the derivation of PMs are presented there. Here, we briefly outline the procedure. The photographic plates were taken at three different epochs: the modern epoch consists of plates taken between 1996 and 2000 with the Las Campanas du Pont $2.5 \mathrm{~m}$ telescope, the intermediate epoch consists of Palomar Observatory Sky Survey plates (POSS-I) taken in the early 1950s with the Palomar Schmidt $1.2 \mathrm{~m}$ telescope, and the old epoch consists of two plates taken in 1909 and 1912 at the Cassegrain focus of the Mount Wilson $1.5 \mathrm{~m}$ telescope. The modern and old plates were digitized with the Yale PDS microdensitometer. Analysis for the POSS-I plates used scans done by both the Space Telescope Science Institute (the Digitized Sky Survey) and the US Naval Observatory, which were then processed at Yale to obtain more accurate positions than provided by the USNO catalogs. Typically, each SA field covers $40^{\prime} \times 40^{\prime}$, an area constrained by the du Pont plates. Two sets of du Pont plates offset by $\sim 20^{\prime}$ in R.A. (to match the Mt. Wilson plate centers) were taken for SA 76 in 1996 and 1998. Each set includes one blue (IIIa-J + GG385) and one visual (IIIa-F + GG495) plate. Three overlapping fields from POSS-I were used, each including one red (103a-D + RP2444) and one blue (103a-O, no filter) plate. PMs were measured separately for the east and west fields of SA 76, as given by the two du Pont sets of plates. Only the du Pont plates were divided into east and west fields-although these east and west plate centers were chosen to match those of the older plates, the coarser plate scale of the Mt. Wilson plates $\left(26 \operatorname{arcsec} \mathrm{mm}^{-1}\right.$ versus $10.9 \operatorname{arcsec} \mathrm{mm}^{-1}$ for the du Pont plates) means that each single Mt. Wilson plate covers the entire area of the combined du Pont fields. The correction to absolute PMs was defined by 97 galaxies in the east field and by 144 galaxies in the west field and applied separately to each field. The uncertainty in the correction to absolute PMs is

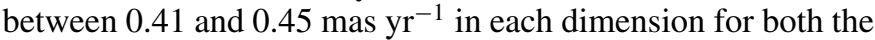
east and west fields. Finally, the two data sets were combined by finding a weighted average absolute PM for well-measured stars in the overlapping region of the two fields. PM uncertainties per


$\sim 4$ mas $\mathrm{yr}^{-1}$ at the faint limit of the survey. The uncertainties are shown as a function of magnitude for all stars in the east and west fields in Figure 1-the solid lines represent a moving median value (in 0.25 mag bins) for PM error as a function of magnitude for each field.

Figure 2 shows absolute PMs in the final combined SA 76 data set for all stars measured on at least four plates spanning at least two of the three widely separated mean epochs. The PMs are divided into a red $(0.9<g-r<1.7)$ and blue $(0.0<g-r<0.9)$ sample to highlight kinematical differences between the predominantly nearby $\mathrm{M}$ dwarfs in the red sample and the more tightly clumped (in PMs) blue sample comprised mostly of thick disk and halo stars (note that the distribution is not centered on, or symmetric about, zero PM-this is mostly due to the Solar motion, and likely also due a contribution from the ACS). By comparison to the SDSS photometric catalog, the PM catalogs are found to be $82 \%$ and $68 \%$ complete at $g=20.0$ for the west and east fields, respectively.

An SDSS color-magnitude diagram (CMD) for all stars with well-measured PMs (i.e., measured on at least five plates from at least two epochs) in SA 76 is seen in Figure 3. The mainsequence turnoff (MSTO) of a distant, metal-poor population is apparent as an overdensity at $g \gtrsim 19,(g-r) \sim 0.2-0.5$, with the main sequence extending down beyond the limits of the survey. The PMs extend $\sim 1.5$ mag fainter than this MSTO feature. 



Figure 2. Proper motions of all stars in SA 76 measured on at least four plates. The upper two panels show proper motions in equatorial coordinates and the bottom panels along Galactic coordinates. These are divided into a red $(0.9<g-r<1.7)$ sample made up of primarily foreground MW dwarfs, and blue $(0.0<g-r<0.9)$ stars, which are mostly MW thick disk and halo stars, along with ACS debris. The more distant thick disk, halo, and ACS stars show more tightly clumped proper motions.

\subsection{Radial Velocities}

\subsubsection{Sample Selection}

As seen in G06, the MSTO feature of the ACS is prominent in SDSS CMDs along the stream. We thus focus our selection of stars for follow-up spectroscopy within the faint $(18.5<$ $g<20.1)$ magnitude and blue $(0.2<g-r<0.6)$ color ranges of the turnoff. As in GCM08, we used preliminary PMs from the CD06 survey to constrain spectroscopic target selection to only those stars within the MSTO region that

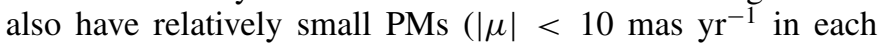
dimension) expected for the distant ACS. Additional targets outside the narrow color, magnitude, and PM selection regions were included at lower priority, in order to fill all of the spectrograph fibers. Furthermore, one fiber configuration of stars at bright magnitudes and colors consistent with the red giant branch (RGB) of the ACS population was observed as a backup target during marginal weather conditions. All targets with spectra having sufficient signal-to-noise that velocities could be derived are shown in the CMD in the left panel of Figure 4, as well as the PM vector point diagram (VPD) of Figure 5 (left panel). Care was taken not to be too restrictive in the color and PM selections, since we had no a priori knowledge of the exact location of ACS debris in either of these dimensions.

\subsubsection{Observations}

RVs were derived from spectroscopic data obtained over the course of four observing runs with the WIYN ${ }^{7} 3.5 \mathrm{~m}$ telescope between December 2006 and November 2008 (Table 1). The Hydra multi-fiber spectrograph was used in two similar configurations. The 2007 February observing run used the 600@10.1 grating in first order with the red fiber cables at a wavelength center of $5400 \AA$, yielding wavelength coverage over $4000-6800 \AA$ at a dispersion of $1.397 \AA$ pixel $^{-1}$, and a spectral resolution of $3.35 \AA$. These data are discussed in GCM08 as the "ACS-C" spectra. During the 2006 December, 2007 December, and 2008 November runs, a similar configuration was utilized (same grating, etc.), but centered slightly redward, providing wavelength coverage $\lambda=4400-7200 \AA$ at the same dispersion and resolution. The general spectral region was selected to include the $\mathrm{H} \beta, \mathrm{Mg}$ triplet, $\mathrm{Na} \mathrm{D}$, and $\mathrm{H} \alpha$ spectral features. We further note

\footnotetext{
7 The WIYN Observatory is a joint facility of the University of Wisconsin-Madison, Indiana University, Yale University, and the National Optical Astronomy Observatory.
} 




Figure 3. SDSS color-magnitude diagram of all stars with well-measured proper motions. The proper-motion catalog is $\gtrsim 68 \%$ complete at $g=20.0$. Anticenter Stream debris is noticeable as an overdensity at faint $(g>19)$, blue $(g-r<0.5)$ colors.

that the 2008 November observing run occurred after the WIYN Bench Spectrograph Upgrade, which included the implementation of a new collimator into the Bench configuration, as well as a new CCD that delivers greatly increased throughput. Each Hydra configuration was exposed multiple times to enable cosmic ray removal. Exposure times, number of stars targeted, and the limiting magnitude of stars in each observed configuration can be found in Table 1 . The 2006 December observations were limited to bright targets due to weather (we were only able to observe briefly between periods of snow and high humidity), while the 2007 February observations were beset by high winds and poor $\left(\sim 2\right.$ '.5) seeing. Good seeing $\left(\sim 00^{\prime} .6\right)$ and clear skies on both the 2007 December and 2008 November observing runs allowed observation of the majority of faint targets within the selection. Typically 60-70 targets were placed on Hydra fibers, with the remaining 15-20 fibers placed on blank sky regions to allow for accurate sky subtraction.

Standard pre-processing was performed on the initial twodimensional spectra using the CCDRED package in IRAF, ${ }^{8}$ and frames were summed before spectrum extraction. Extraction of one-dimensional spectra and further spectroscopic reduction used the DOHYDRA utilities (also in IRAF). CuAr arc lamp exposures were taken at each configuration; from these, 30-35

\footnotetext{
8 IRAF is distributed by the National Optical Astronomy Observatory, which is operated by the Association of Universities for Research in Astronomy
} (AURA), under cooperative agreement with the National Science Foundation.
Table 1

Summary of WIYN+Hydra Spectroscopic Observations in SA 76

\begin{tabular}{lccc}
\hline \hline \multicolumn{1}{c}{ Date } & $\begin{array}{c}\text { Exposures } \\
(\mathrm{s})\end{array}$ & $N_{\text {stars }}$ & Mag. Limit $^{\mathrm{a}}$ \\
\hline Dec 2006 & $3 \times 1800$ & 66 & 18.5 \\
Feb $2007^{\mathrm{b}}$ & $9 \times 2400$ & 61 & 20.1 \\
Dec 2007 & $6 \times 2700$ & 57 & 20.0 \\
Nov 2008 & $8 \times 1800$ & 59 & 20.1 \\
TOTAL & & $224^{\mathrm{c}}$ & \\
\hline
\end{tabular}

Notes.

a SDSS $g$ magnitudes.

b These observations were reported in GCM08 as their field "ACS-C."

${ }^{c}$ Total number is less than the sum of targets from individual runs because some stars were observed on multiple runs.

emission lines were used to fit the dispersion solution for each Hydra configuration. A few RV standards were targeted on each observing run covering spectral types from $\mathrm{F}$ through early $\mathrm{K}$ (both dwarfs and giants), each through multiple fibers, to yield multiple individual cross-correlation template spectra. These RV standard spectra were first cross-correlated against each other using the IRAF tool FXCOR to determine the accuracy of the velocities and remove any outliers (i.e., those that yield unreasonable cross-correlation results due to some defect, such as a poorly removed cosmic ray). Measured velocities of the RV standards typically agreed with published IAU standard values to within 1-2 $\mathrm{km} \mathrm{s}^{-1}$. RVs for program stars were derived by cross-correlating all object spectra against all of the standards taken on the same observing run. To maximize the cross-correlation signal-to-noise ratio $(\mathrm{S} / \mathrm{N})$ in faint, metalpoor stars, only the regions around the $\mathrm{H} \beta, \mathrm{Mg}$ triplet, and $\mathrm{H} \alpha$ absorption lines were used for cross-correlation.

RV uncertainties were derived using the Vogt et al. (1995) method, as described in Muñoz et al. (2006) and Frinchaboy et al. (2006). The Tonry-Davis ratio (TDR; Tonry \& Davis 1979) scales with $\mathrm{S} / \mathrm{N}$, such that individual RV errors can be calculated directly from the TDR, provided you have multiple observations of some particular standard star to map the dependence. Where possible, we have used this technique, but for the 2006 December observing run, only a total of four RV standard spectra were taken. Thus, the RV uncertainty for the 2006 December object stars is the standard deviation of the results from crosscorrelation against these four standards. Typical RV uncertainties for individual measurements were $\sigma_{V} \approx 5-10 \mathrm{~km} \mathrm{~s}^{-1}$, with most spectra having $\mathrm{S} / \mathrm{N} \sim 15-20$. From repeat measures of a handful of stars, we found mean systematic offsets of $\sim 5-8 \mathrm{~km} \mathrm{~s}^{-1}$ between observing runs. These offsets were applied to all RVs from a given run to place all measurements on the system of the 2007 December velocities.

\section{ACS CANDIDATE SELECTION}

The upper panel of Figure 6 shows measured heliocentric RVs for all 224 stars in the SA 76 sample. As already shown by GCM08, the distribution has multiple velocity peaks-one broad and prominent peak at $\sim 30-40 \mathrm{~km} \mathrm{~s}^{-1}$ associated with Galactic stellar populations and another at $V_{\text {helio }} \sim 90 \mathrm{~km} \mathrm{~s}^{-1}$ identified as ACS debris. In the lower panel of Figure 6, velocities are shown for only faint $(g>18.5)$ stars, in order to focus on the MSTO region of the ACS. To facilitate comparisons to expected Galactic populations in this work, we combined five realizations of the Besançon Galaxy model (Robin et al. 2003) along this same line of sight. Combining several model queries 


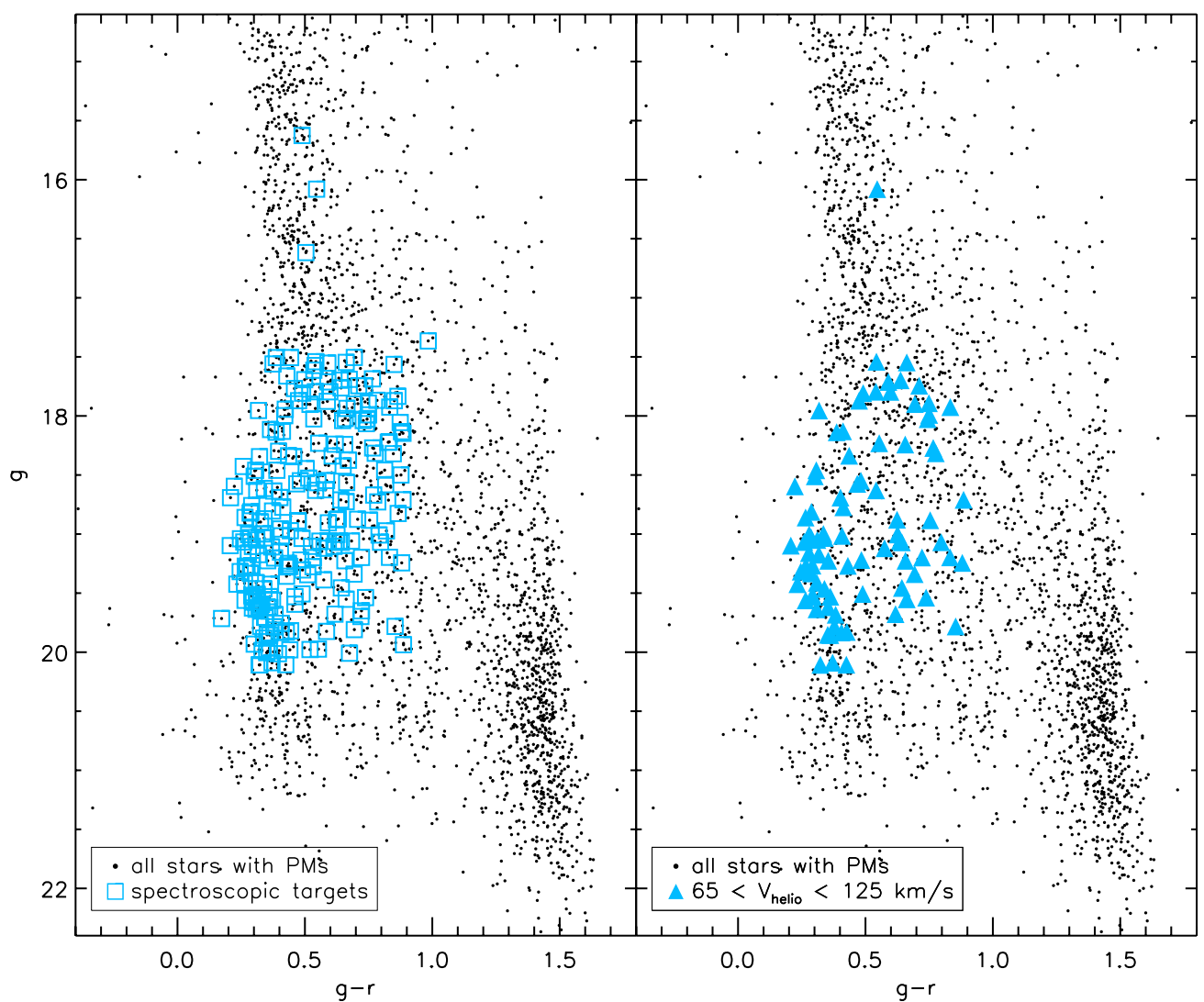

Figure 4. Color-magnitude diagrams of all stars with well-measured proper motions, highlighting the spectroscopically observed samples. Left panel: open squares show all stars observed spectroscopically (see Section 2.1 for details on target selection). Right panel: solid triangles represent stars within the initial $65 \mathrm{~km} \mathrm{~s}^{-1}<V_{\text {helio }}<$ $125 \mathrm{~km} \mathrm{~s}^{-1}$ velocity selection discussed in Section 3. The majority of these selected candidates are confined to an apparent upper main sequence at $g>18.5$, $0.2<(g-r)<0.5$.

(A color version of this figure is available in the online journal.)

serves to smooth over finite sampling statistics from within each model population and reduce the noise in the predicted distributions. For comparison to our measured RVs, we overlay in Figure 6 (as a filled histogram) an RV distribution taken from the combined realizations of the Besançon Galaxy model, scaled to match the total number of stars observed. The model distribution was limited to the magnitude and color ranges of our target selection $(0.2<g-r<0.6$ and $18.5<g<20.0)$ to sample the same foreground/background populations (we note that this is the same comparison done by GCM08, but with additional measured RVs). The peak and dispersion of the observed RVs match the model distribution fairly well, but with an additional peak prominently visible at $V_{\text {helio }} \sim 90 \mathrm{~km} \mathrm{~s}^{-1}$. This peak has already been identified by GCM08 (though using far fewer RV measurements) as being due to ACS stream stars.

ACS candidates are initially selected using all stars with $65 \mathrm{~km} \mathrm{~s}^{-1}<V_{\text {helio }}<125 \mathrm{~km} \mathrm{~s}^{-1}$ (to ensure that all possible stream members are included) - a total of 87 stars. Stars within this RV selection are shown as solid triangles in the right-hand panels of Figures 4 and 5. Many of the stars thus selected are concentrated along the apparent main sequence of the ACS population in the SDSS CMD and are also more tightly clumped in the VPD than the general Galactic populations. Because the ACS velocity peak overlaps the Galactic distribution, however, a selection of ACS candidates based solely on RVs will contain some contamination from foreground (and background) MW stars. Initially, we remove all stars having PMs $|\mu| \gtrsim$ 10 mas $\mathrm{yr}^{-1}$ in either dimension. Such PMs imply extremely large ( $\gtrsim 500 \mathrm{~km} \mathrm{~s}^{-1}$ ) tangential velocities if these stars are at distances $\sim 10 \mathrm{kpc}$. Thus most of the stars removed on this basis are nearby, high PM MW disk stars.

Examination of the remaining sample of stars in a CMD (right panel of Figure 4) shows no obvious collection of bright $(g<$ 18.5) stars with a narrowly defined RGB locus representing an evolved counterpart to the well-defined MSTO. Therefore, to disentangle comoving stream members from foreground/ background MW stars, we turn to the reduced proper motion diagram (RPMD). Reduced PM, first used extensively by Luyten (e.g., Luyten 1922), is defined as $H_{m} \equiv m+5 \log \mu+5$, where $m$ is apparent magnitude in a given bandpass (we will use SDSS $g$ magnitudes hereafter) and $\mu$ is the total PM in $\operatorname{arcsec} \mathrm{yr}^{-1}$. Because the tangential velocity $V_{\tan }\left(\mathrm{km} \mathrm{s}^{-1}\right)=4.74 \times d \times \mu$ (where $d$ is in pc and $\mu$ in $\operatorname{arcsec} \mathrm{yr}^{-1}$ ), the reduced PM $H$ is analogous to an absolute magnitude for stars of a common motion and virtually independent of distance. Substituting terms and using the definition of absolute $g$ magnitude, one finds that $H_{g}=M_{g}+5 \log V_{\tan }-3.38$. Since we know the expected color-magnitude locus for a given population, the reduced PM diagram $\left(H_{g}\right.$ versus $\left.g-r\right)$ can be used to identify objects with similar tangential velocities. The RPMD for all objects measured on at least five plates (to ensure well-measured PMs) is shown in Figure 7. The left panel shows all well-measured objects. In the right panel, all spectroscopic targets are depicted with open (gray) squares, with solid symbols representing stars within the initial RV selection. Stars in the RV-selected sample with red $(0.5<g-r<0.9)$ colors and having 

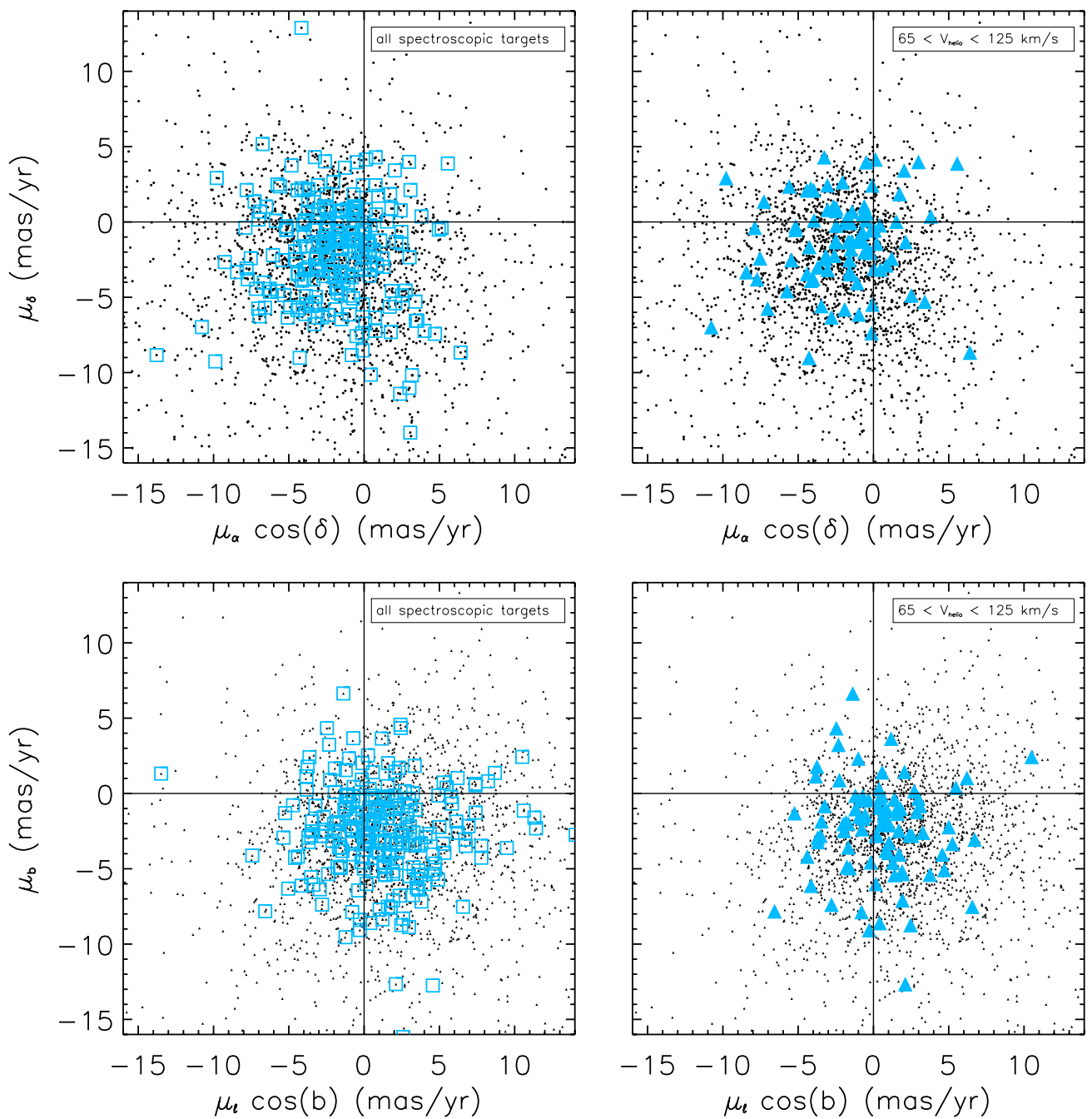

Figure 5. All well-measured proper motions in SA 76, highlighting the same spectroscopically observed samples as Figure 4. Left panel: open squares show all stars observed spectroscopically. Right panel: solid triangles represent stars within the initial $65 \mathrm{~km} \mathrm{~s}^{-1}<V_{\text {helio }}<125 \mathrm{~km} \mathrm{~s}^{-1}$ velocity selection. Most candidates thus selected are clumped more tightly than the overall spectroscopic sample.

(A color version of this figure is available in the online journal.)

reduced PM inconsistent with that expected for the ACS main sequence and giant branch were first removed from the sample; these are depicted as filled green triangles in Figure 7. It is apparent in this plot that the remaining RV-selected candidates separate into two groupings that resemble MSTO features at blue $(0.2<g-r<0.5)$ colors at reduced PMs of $11 \lesssim H_{g} \lesssim$ 12.5 and $12.5 \lesssim H_{g} \lesssim 14.5$. These groups were separated into a "lower" (shown as solid blue squares in Figures 7 and 8) and an "upper" (filled red diamonds) RPMD sample, and examined to see whether there are two populations at distinct $V_{\text {tan }}$ within our RV selection. The upper RPMD sample is tightly clumped in the PM VPD (Figure 8, red filled squares), while the lower RPMD sample shows considerable scatter inconsistent with a common-motion stellar population at $10 \mathrm{kpc}$. Because of the wide dispersion in their PMs, we surmise that the lower RPMD sample is neither ACS debris nor any comoving, spatially localized structure; we thus remove all of these stars from the sample of ACS candidates.

Among this sample (red filled diamonds in Figure 8) there remain some outliers well outside the PM clump. These were examined to assess their membership in the stream. PMs in equatorial coordinates are shown for the entire RV-selected sample as a function of $g$ magnitude and $g-r$ color in Figure 9. If the brightest, reddest stars in the initial sample selection are red giants or subgiants associated with the ACS, they should have tightly clumped PMs at the same mean PM as the MSTO stars (especially when one considers that the brighter stars should have more precise PM measurements). The contrary is true, however-bright, red stars exhibit much more scatter in both PM dimensions than the tightly clumped faint mainsequence stars. Because this work is focused on measuring the mean motion of the stream, and not necessarily identifying every possible member, and because no obvious RGB population of the ACS is seen, all stars with $g<18.5$ were removed from the final set of PM candidates. To retain only well-measured stars, those stars measured on fewer than five of the 15 plates were also culled from the sample, leaving a total of 31 rather secure ACS members, based on all available information. These are confined to a thin, well-defined MSTO in the CMD of Figure 10 (left panel), which corresponds to the obvious main sequence visible in the right panel. This panel shows all stars from SDSS DR7 in this field of view, with two isochrones from Dotter et al. (2008) for old $(10 \mathrm{Gyr})$, metal-poor $([\mathrm{Fe} / \mathrm{H}]=-0.9$ and $[\mathrm{Fe} / \mathrm{H}]=-1.3$; chosen to be close to the median metallicities 


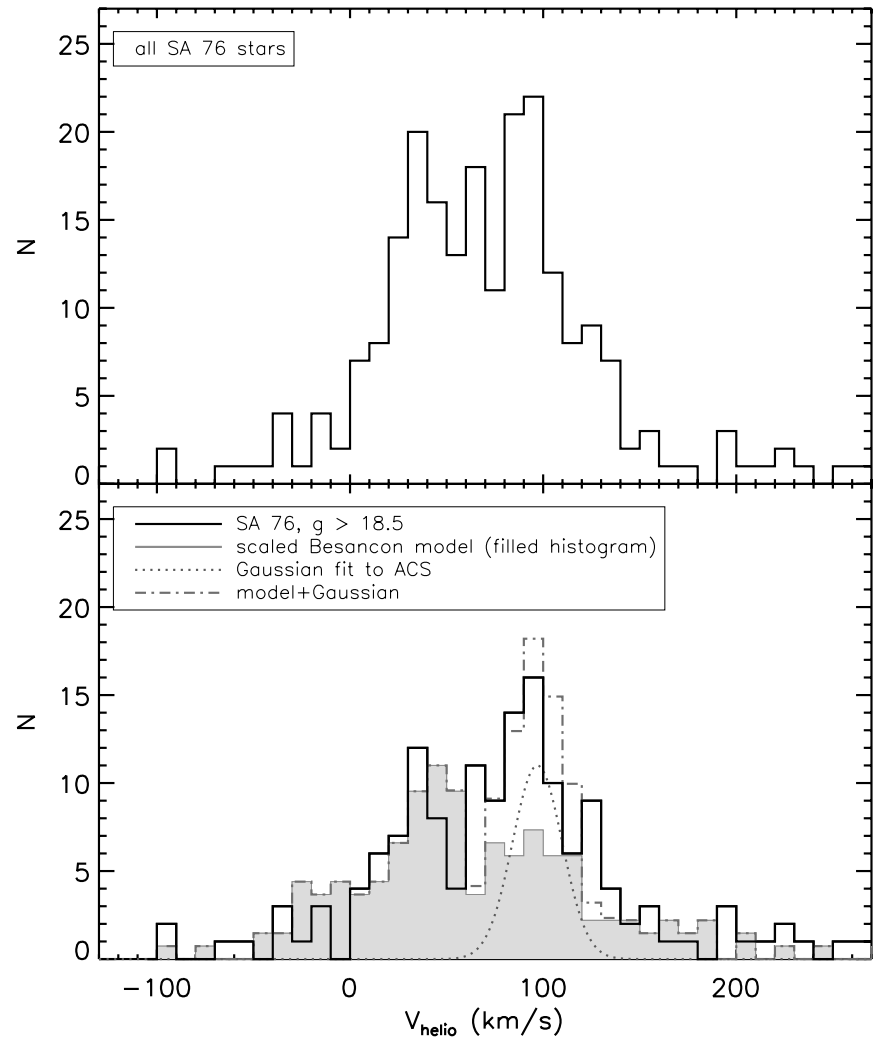

Figure 6. Upper panel: measured heliocentric radial velocities of all observed targets in SA 76. Two prominent peaks are visible at $V_{\text {helio }} \sim 35 \mathrm{~km} \mathrm{~s}^{-1}$ and $V_{\text {helio }} \sim 90 \mathrm{~km} \mathrm{~s}^{-1}$. Lower panel: measured heliocentric velocities for all targets in SA 76 having magnitudes $g>18.5$. The filled histogram shows the expected velocities from a scaled sum of five Besançon model queries along the SA 76 line of sight, selected within the same magnitude and color ranges as the ACS candidates. A Gaussian with the maximum likelihood results for the mean velocity $\left(V_{\text {helio }}=97.0 \mathrm{~km} \mathrm{~s}^{-1}\right)$ and dispersion $\left(\sigma_{0}=12.8 \mathrm{~km} \mathrm{~s}^{-1}\right)$ of the Anticenter Stream is overplotted as a dotted curve. The sum of the Besançon model (representing Galactic stellar populations, and scaled so that the final dot-dashed histogram matches the number of stars observed spectroscopically) and the best-fit Gaussian (i.e., ACS members) is shown as a dot-dashed histogram.

found for ACS members in Section 4) populations at $10 \mathrm{kpc}$, the distance we adopt for the ACS. The isochrones were also corrected for the mean reddening $E(B-V)=0.04$ (Schlegel et al. 1998) in the SA 76 field. These ridgelines follow the clear overdensity of faint, blue stars, as well as passing through the MSTO locus defined by the final sample of identified ACS members. The ACS members also clump tightly in the PM VPD (Figure 11), as expected for a distant, comoving stellar population.

\section{METALLICITIES}

Stellar parameters are estimated for all spectroscopic targets using a software pipeline developed by author J.L.C. and others in the University of Virginia stellar populations research group for this purpose. This pipeline uses fits to the distribution of $[\mathrm{Fe} / \mathrm{H}]$ as a function of low-resolution Lick Fe, $\mathrm{Mg}$, and $\mathrm{H} \beta$ indices from the library of Schiavon (2007, based on the spectra of Jones 1998) to determine individual stellar metallicities. Details about the code, which is called EZ_SPAM (Easy Stellar Parameters and Metallicities), will be found in a forthcoming paper (J. L. Carlin et al. 2011, in preparation).

Measured metallicities for all stars observed in SA 76 are shown in the upper panel of Figure 12. The expected distribution (scaled to match the number of observed stars) from the Besançon galaxy model for stars with similar color and magnitude distribution as our targets is shown for comparison (as we did for the RV sample). The distributions match quite well on visual inspection, and a two-sided Kolmogorov-Smirnov (K-S) test finds a 79\% probability that the two samples are drawn from the same parent population (we note that this would likely be higher if a number of these stars were not ACS members). The lower panel of Figure 12 shows $[\mathrm{Fe} / \mathrm{H}]$ for only the 31 stars identified as ACS members (dashed histogram), again overlaying the scaled Besançon distribution (dot-dashed lines) for corresponding selection criteria. The median metallicity of the ACS members is $[\mathrm{Fe} / \mathrm{H}]=-0.81$, but with significant scatter. The median for the model is $[\mathrm{Fe} / \mathrm{H}]=-0.91$, and the K-S test for these two samples suggests a $47 \%$ probability that the ACS members are drawn from the same population as the model distribution. However, only 9 of these 31 spectra have $\mathrm{S} / \mathrm{N}>20$, so most of these measurements of $[\mathrm{Fe} / \mathrm{H}]$ should be regarded with some skepticism. The nine stars with well-measured metallicities (seen as a solid, filled histogram in Figure 12) yield median $[\mathrm{Fe} / \mathrm{H}]=-1.26$ and include two stars with $[\mathrm{Fe} / \mathrm{H}] \lesssim-1.9$. Again, the spread in metallicity is rather broad, as is seen in many dSph systems, where there are often multiple epochs of star formation.

The apparent agreement between metallicities of the overall ACS member sample with the Besançon predictions (if real; see the caveat regarding low $\mathrm{S} / \mathrm{N}$ of most of these spectra in the previous paragraph) could arise for several reasons. It may be that the ACS "members" are actually a sample of thick disk and/or halo stars (noting that, at $b=26.4$, or $Z_{\mathrm{GC}}=4.4 \mathrm{kpc}$ at a distance of $10 \mathrm{kpc}$, near the Galactic anticenter, the line of sight toward SA 76 is not sampling many thin disk MSTO stars at faint magnitudes), and thus agree with the $[\mathrm{Fe} / \mathrm{H}]$ expected for smooth Galactic populations because they are drawn from exactly those populations. From the five realizations of the Besançon model used for comparison, we find that a total of $214 \pm 13$ stars are expected within the color-magnitude selection initially applied $(0.2<g-r<0.6$ and $18.5<$ $g<20.0$ ). Of these, only $4 \pm 2$ are thin-disk stars, with the rest made up of thick disk and halo populations. In SA 76, we find 271 stars within the same selection criteria (in a region of equal area to that of the model), a significant $(\sim 4 \sigma)$ excess over the number of stars expected from the star-count models of smooth Galactic populations that contribute to the Besançon model. Moreover, the ACS shows a well-defined narrow main sequence in the SDSS CMD of Figure 10 (or, more clearly, in Figure 3 of G06) indicative of a limited radial extent, unlike the smoother and more broadly distributed stellar populations that may arise if the ACS results from a warp or flare of the Galactic disk or a flyby encounter with a massive perturber (Kazantzidis et al. 2008; Younger et al. 2008). Furthermore, if the line of sight intersected a warped or flared MW disk, it is unlikely that we would find the clear spatial separation of the narrow ACS stream from the Galactic thin/thick disks seen in G06 (see also our Figure 14 below). It is thus unlikely that the ACS sample consists primarily of the nominal MW stellar populations. The second (and more likely) possibility is that the ACS stars are metal-poor remnants of a disrupted $\mathrm{dSph}$, and thus resemble the metal-poor MW halo (thought to be comprised mostly of stars from tidally disrupted dSphs and globular clusters; e.g., Majewski et al. 1994; Bullock \& Johnston 2005) in the same magnitude and color ranges. Discrimination between these two scenarios could be provided by high-resolution 


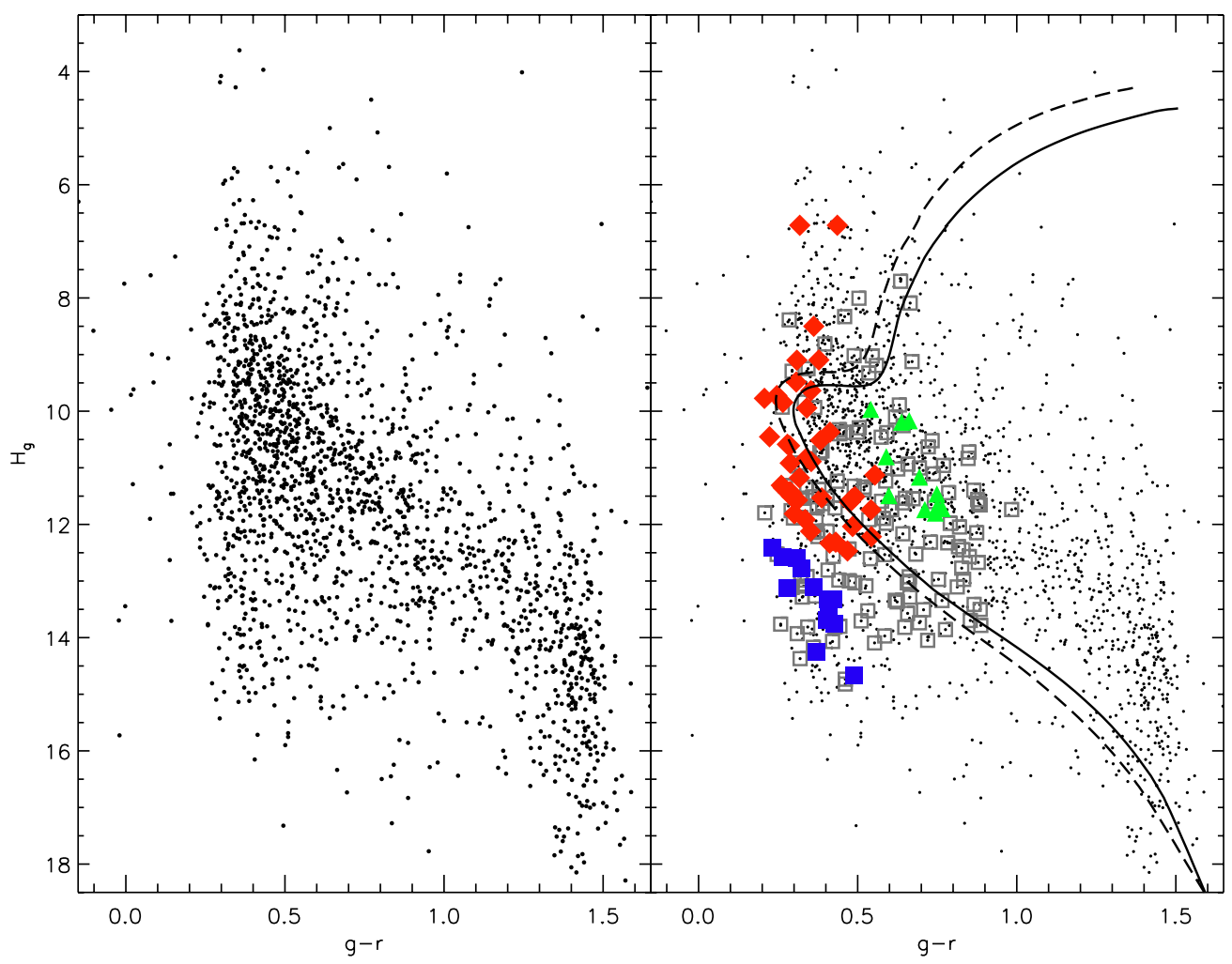

Figure 7. Reduced proper motion diagram (RPMD) for all well-measured stars in SA 76, where $H_{g} \equiv g+5 \log \mu+5$. The left panel shows all well-measured objects. The swath of stars running across the diagram from $g-r \sim 0.4, H_{g} \sim 9$ to $g-r \sim 1.4, H_{g} \sim 15$ consists mainly of nearby MW disk stars, with the vertical feature at $g-r \sim 1.4$ made up of local M dwarfs. The extension to higher values of $H_{g}$ at $g-r \sim 0.4$ (i.e., below the disk stars) represents either a population at larger tangential velocity than the disk stars or metal-poor Galactic subdwarfs. In the right panel, all spectroscopic targets are depicted with open (gray) squares, with solid symbols representing stars within the initial RV selection $65 \mathrm{~km} \mathrm{~s}^{-1}<V_{\text {helio }}<125 \mathrm{~km} \mathrm{~s}^{-1}$. For reference, two 10 Gyr ridgelines from Dotter et al. (2008) at a distance of $10 \mathrm{kpc}$, with a tangential velocity of $70 \mathrm{~km} \mathrm{~s}^{-1}$, and $[\mathrm{Fe} / \mathrm{H}]=-1.3$ and -0.9 (solid and dashed lines, respectively) are shown. These ridgelines have also been corrected for the mean reddening $(E(B-V)=0.04$ according to the Schlegel et al. 1998 maps) along the line of sight. Stars in the RV-selected sample at red $(0.5<g-r<0.9)$ colors having $H_{g}$ inconsistent with the expected ACS main sequence and giant branch were removed from the sample; these are shown as filled green triangles. The remaining RV-selected candidates were separated into two groupings that resemble MSTO features at blue $(0.2<g-r<0.5)$ colors and reduced PMs of $11 \lesssim H_{g} \lesssim 12.5$ and $12.5 \lesssim H_{g} \lesssim 14.5-$ a "lower" (solid blue squares) and an "upper" (filled red diamonds) sample. These two samples were examined to determine whether there are two distinct turnoffs at different tangential velocities in SA 76 (and within the RV selection). The lower sample (blue squares) apparently consists of foreground (or background) contamination by MW halo subdwarfs and was removed from the final ACS sample.

(A color version of this figure is available in the online journal.)

spectroscopic abundance analysis, wherein unique abundance patterns may be able to distinguish differences between the populations.

\section{ANTICENTER STREAM KINEMATICS}

\subsection{Radial Velocities}

From the final selected ACS candidates, kinematical properties of the ACS in SA 76 were estimated using a maximum likelihood method (e.g., Pryor \& Meylan 1993; Hargreaves et al. 1994; Kleyna et al. 2002). A systemic heliocentric RV of $V_{\text {helio }}=97.0 \pm 2.8 \mathrm{~km} \mathrm{~s}^{-1}$ was derived for the ACS component, with an intrinsic dispersion $\sigma_{0}=12.8 \pm 2.1 \mathrm{~km} \mathrm{~s}^{-1}$. A Gaussian centered at this velocity, with FWHM matching the measured dispersion, and encompassing the total number of ACS candidates is overplotted as a dotted curve on the RV histogram in the lower panel of Figure 6. The sum of this Gaussian distribution and the scaled, binned Besançon model distribution (filled histogram) is plotted as the dot-dashed histogram, which reproduces the observed velocities remarkably well. This confirms our interpretation of the $\sim 97 \mathrm{~km} \mathrm{~s}^{-1}$ peak, which is not expected among smooth Galactic populations, as being due to kinematically cold substructure. The measured $V_{\text {helio }}$ agrees with the measurement $\left(V_{\text {helio }}=88.8 \pm 5.0 \mathrm{~km} \mathrm{~s}^{-1}\right)$ of GCM08 at the $1.6 \sigma$ level (though we remind the reader that those same data are also included in our sample here), but we derive a dispersion more than twice the $\sigma_{0}=5.9 \mathrm{~km} \mathrm{~s}^{-1}$ found by GCM08. This may mean that GCM08 underestimated the intrinsic dispersion of the ACS from their limited data set, or it may be that GCM08 sampled one of the cold "tributaries" found by G06 to make up the larger ACS. In this latter scenario, the superposition of multiple cold populations (whether from tributaries or multiple orbital wraps) would lead to an overall larger measured dispersion. Whichever of these is the case, our measured $\sigma_{0}=12.8 \mathrm{~km} \mathrm{~s}^{-1}$ is typical of a tidal stream from a disrupted dwarf galaxy (e.g., Majewski et al. 2004; Monaco et al. 2007); further high-resolution study of large numbers of stars would be necessary to explore the possibility of multiple stream tributaries within this field of view. We also note that the $\sigma_{0}=12.8 \mathrm{~km} \mathrm{~s}^{-1}$ dispersion we measure in SA 76 is consistent with result $\left(\sigma_{0} \sim 14.9 \mathrm{~km} \mathrm{~s}^{-1}\right)$ found by GCM08 for ACS debris $\sim 23^{\circ}$ from SA 76 in their field "ACS-B" at $(\alpha, \delta)_{2000} \sim\left(124^{\circ}, 37^{\circ}\right)$.

\subsection{Proper Motions}

From this same sample of 31 ACS candidates, maximum likelihood estimates were derived for the absolute PMs of $\mu_{\alpha} \cos$ 


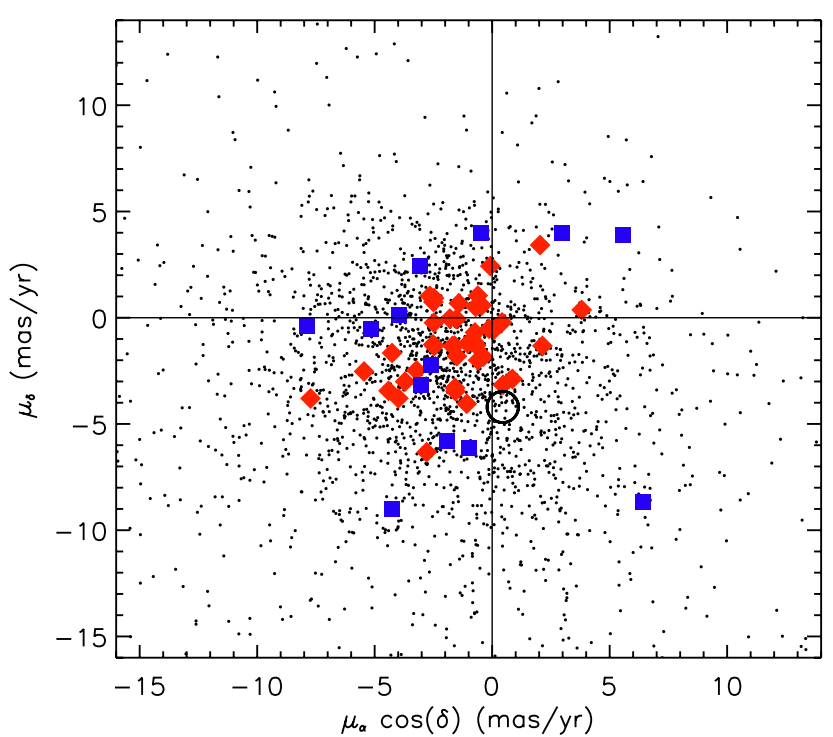

Figure 8. Vector point diagram of all well-measured stars in SA 76. The upper and lower RPMD samples (as defined in Figure 7) are shown as red diamonds and blue squares, respectively. The red diamonds (upper RPMD sample) clump tightly in proper motions, as expected for a distant, commonmotion stellar population. The lower RPMD sample (blue squares) shows large scatter, suggesting they are Milky Way stars (likely metal-poor halo subdwarfs) in the foreground (or background) of the ACS rather than a coherently moving stellar population. To select a relatively "pure" ACS sample, we removed all "lower RPMD" stars. The open circle represents the solar reflex motion for an object at $10 \mathrm{kpc}$ (i.e., the heliocentric proper motion one would measure for an object stationary with respect to the Galaxy) along the SA 76 line of sight.

(A color version of this figure is available in the online journal.)

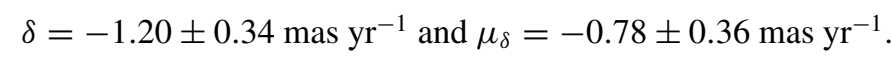
The uncertainties of individual stars used in these estimates contain the error in the PM zero point (derived from galaxies and QSOs) added in quadrature to the measurement uncertainty of each individual relative PM. Both of these PM measurements differ significantly from those of GCM08; our $\mu_{\alpha} \cos \delta$ is

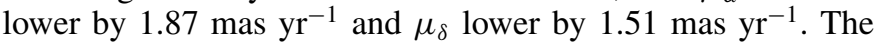
GCM08 estimate was based on 16 RV members, with PMs from SDSS/USNO-B (Munn et al. 2008, 2004). The SDSS/ USNO-B PMs have individual uncertainties of $\sim 4$ mas $\mathrm{yr}^{-1}$, so we regard the GCM08 estimates as only rough limits on the tangential motions of the stream. Our current absolute PMs are derived from much more precise (1-2 mas $\mathrm{yr}^{-1}$ per star) PMs, as well as a larger number of securely identified members; we thus consider these new estimates more reliable. We note, however, that although the components of the implied space velocities from our measurements differ significantly from those of GCM08 (mostly in the component vertical to the disk), the magnitude of the motion differs by only $\sim 40 \mathrm{~km} \mathrm{~s}^{-1}$ (assuming a $10 \mathrm{kpc}$ distance). Converted to PMs along Galactic coordinates, our results become $\mu_{l} \cos b=0.23 \pm 0.36{\text { mas } \mathrm{yr}^{-1} \text { and }}^{-1}$

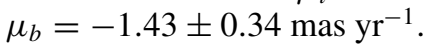

\subsection{Three-dimensional Motion}

The measurements of all three components of stream star motions in SA 76 were used to estimate the orbital characteristics of the ACS. Some information about the stream distance is needed to convert position on the sky to a Galactocentric position. Grillmair (2006) estimated the distance to the ACS to
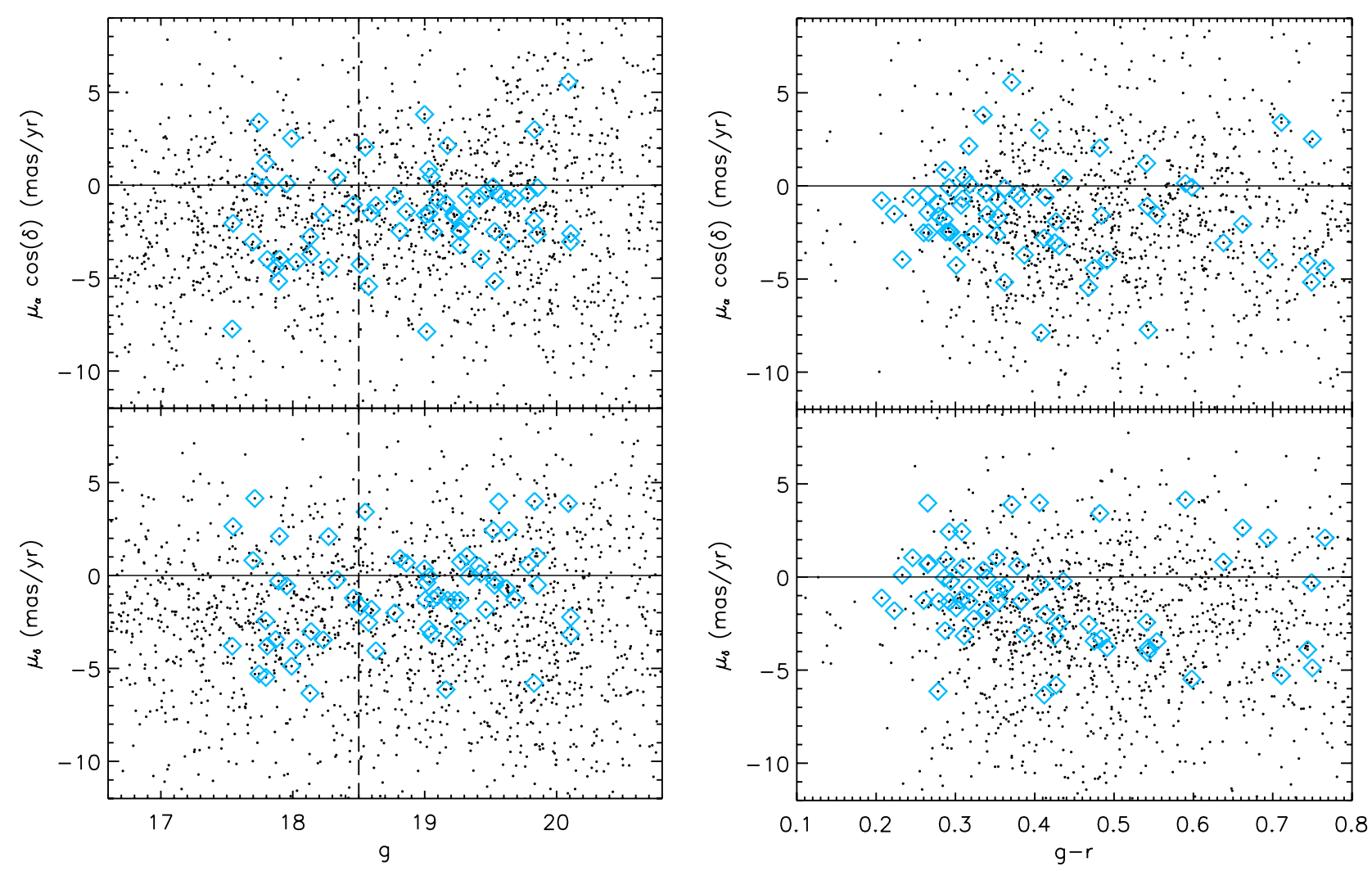

Figure 9. Proper motions of all stars measured on at least four plates (to remove spurious detections) as a function of $g$ magnitude (left) and $g-r$ color (right). Open (blue) diamonds are those stars within our initial ACS candidate selection $\left(65 \mathrm{~km} \mathrm{~s}^{-1}<V_{\text {helio }}<125 \mathrm{~km} \mathrm{~s}^{-1}\right)$. Bright $(g<18.5)$, predominantly red $(g-r \gtrsim 0.5)$ stars exhibit $\sim 1.5 \times$ (in rms) larger scatter in both proper motion dimensions than the fainter ACS main-sequence candidates. Because we are interested in the mean ACS motion, and not necessarily identifying all ACS members in SA 76, we chose to remove bright stars from the ACS candidates. The vertical line at $g=18.5$ marks the magnitude cut performed (in addition to the RPMD criteria discussed in Section 3) in selecting our final ACS sample.

(A color version of this figure is available in the online journal.) 


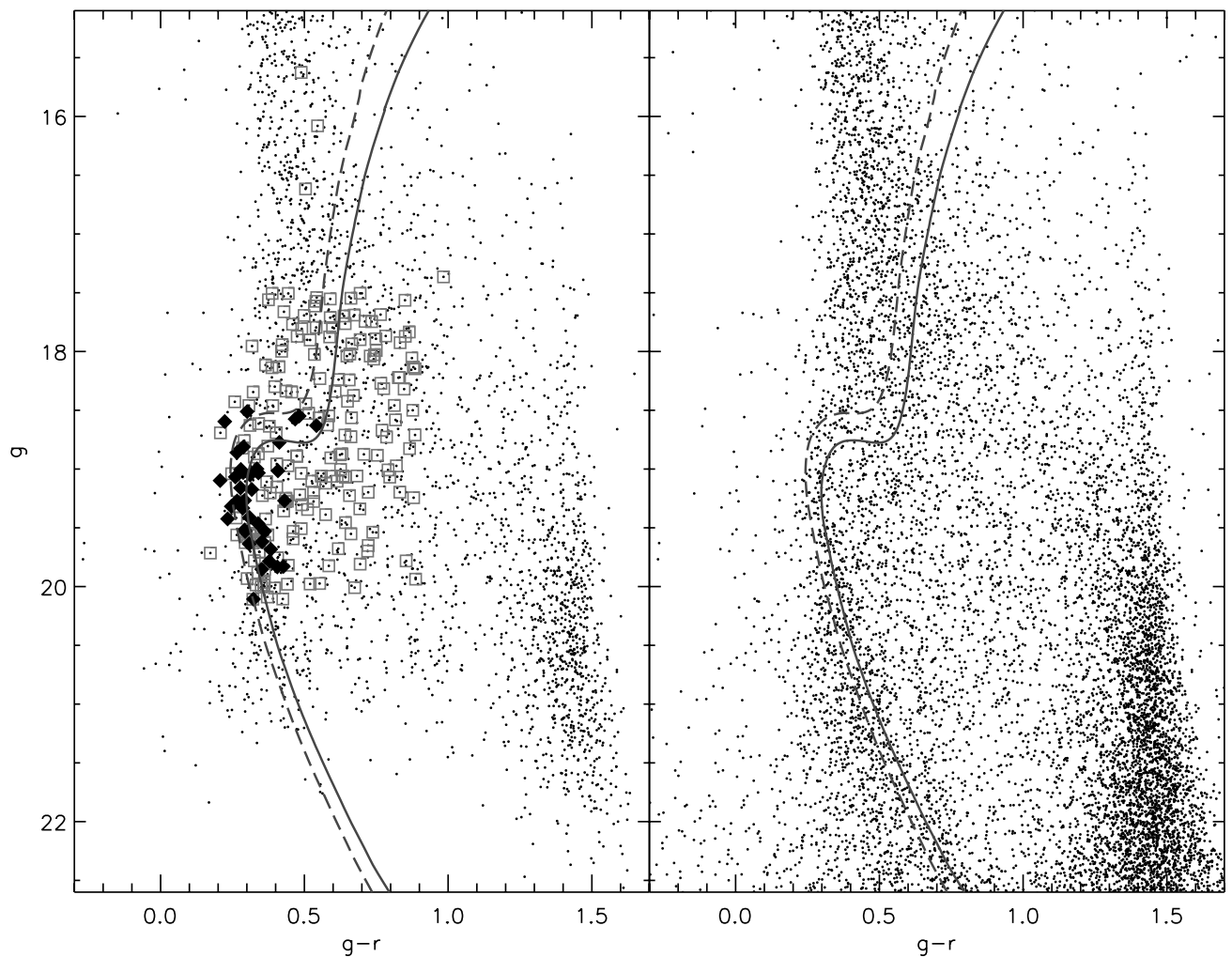

Figure 10. Left panel: color-magnitude diagram of all stars in the proper motion catalog (dots) with spectroscopic targets shown as open squares. The final ACS sample of 31 candidates is represented by filled diamonds. For comparison, two isochrones from Dotter et al. (2008) for old (10 Gyr) populations at a distance of $10 \mathrm{kpc}$ are shown: the solid line has $[\mathrm{Fe} / \mathrm{H}]=-0.9$ and the dashed line $[\mathrm{Fe} / \mathrm{H}]=-1.3$. Both isochrones have been corrected for the mean reddening of $E(B-V)=0.04$ from Schlegel et al. (1998) for this line of sight. Right panel: all objects classified as stars in SDSS DR7, in a 1.3 × 1.3 region centered on SA 76. The ridgelines are the same as in the left panel. The main sequence of the Anticenter Stream can be seen clearly among faint, blue stars.

Table 2

Anticenter Stream Kinematics in SA $76^{\mathrm{a}}$

\begin{tabular}{|c|c|c|c|c|c|c|c|c|c|}
\hline $\begin{array}{c}V_{\text {helio }} \\
\left(\mathrm{km} \mathrm{s}^{-1}\right) \\
\end{array}$ & $\begin{array}{c}V_{\mathrm{GSR}} \\
\left(\mathrm{km} \mathrm{s}^{-1}\right)\end{array}$ & $\begin{array}{c}\sigma_{0} \\
\left(\mathrm{~km} \mathrm{~s}^{-1}\right)\end{array}$ & $\begin{array}{l}\mu_{\alpha} \cos (\delta)^{\mathrm{b}} \\
\left(\mathrm{mas}_{\mathrm{yr}}^{-1}\right)\end{array}$ & $\begin{array}{c}\mu_{\delta} \\
\left(\operatorname{mas~yr}^{-1}\right)\end{array}$ & $\begin{array}{c}\mu_{l} \cos b \\
\left(\mathrm{mas} \mathrm{yr}^{-1}\right)\end{array}$ & $\begin{array}{c}\mu_{b} \\
\left(\operatorname{mas~yr}^{-1}\right)\end{array}$ & $\begin{array}{c}U_{\mathrm{GSR}^{\mathrm{c}}} \\
\left(\mathrm{km} \mathrm{s}^{-1}\right)\end{array}$ & $\begin{array}{c}V_{\mathrm{GSR}} \\
\left(\mathrm{km} \mathrm{s}^{-1}\right)\end{array}$ & $\begin{array}{c}W_{\mathrm{GSR}} \\
\left(\mathrm{km} \mathrm{s}^{-1}\right)\end{array}$ \\
\hline $97.0 \pm 2.8$ & $-6.3 \pm 2.8$ & $12.8 \pm 2.1$ & $-1.20 \pm 0.34$ & $-0.78 \pm 0.36$ & $0.23 \pm 0.36$ & $-1.43 \pm 0.34$ & $-86.7 \pm 12.2$ & $158.5 \pm 17.0$ & $-10.5 \pm 23.4$ \\
\hline
\end{tabular}

Notes. All calculations assume $d_{\mathrm{ACS}}=10.0 \mathrm{kpc}$, and $V_{\mathrm{circ}}=220 \mathrm{~km} \mathrm{~s}^{-1}$ at $R_{0}=8.5 \mathrm{kpc}$. This yields right-handed, Galactocentric coordinates for this field of $(X, Y, Z)_{\mathrm{GC}}=(-16.32,-4.38,4.44) \mathrm{kpc}$.

a $(\alpha, \delta)_{2000}=(125.28,14.69) ;(l, b)=(209.25,26.36)$

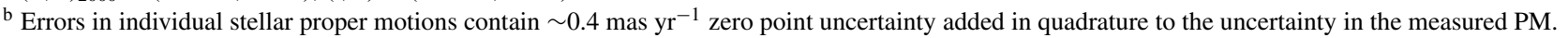

${ }^{c}$ We used the Dehnen \& Binney 1998 values for the solar peculiar motion: $\left(U_{0}, V_{0}, W_{0}\right)=(10.00,5.25,7.17) \pm(0.36,0.62,0.38) \mathrm{km} \mathrm{s}^{-1}$ (in a right-handed frame).

be $8.9 \pm 0.2 \mathrm{kpc}$, based on the required shift of an M13 fiducial to match the ACS main sequence. Though this provided a peak in the matched filtering, the metallicity of $\mathrm{M} 13([\mathrm{Fe} / \mathrm{H}]=$ -1.54 ; Harris 1996) is lower than the median we measured of $[\mathrm{Fe} / \mathrm{H}] \approx-0.8$ for ACS members $([\mathrm{Fe} / \mathrm{H}] \approx-1.3$ for the nine high $\mathrm{S} / \mathrm{N}$ members; see Section 4 for details). We suggest that a slightly more metal-rich isochrone than that of M13 would be a more reasonable fit. This would place the stream stars slightly farther away than the $\sim 9 \mathrm{kpc}$ from G06. Given the uncertainty in the stream distance, we adopt $10 \pm 3 \mathrm{kpc}$ for the distance to the ACS in SA 76 in all further calculations in this work.

The prescription of Johnson \& Soderblom (1987) was used to derive the Cartesian Galactic space velocity components $(U, V$, and $W)$ in a right-handed frame with the origin at the Galactic center (i.e., $U$ is positive toward the Galactic center, $V$ in the direction of Galactic rotation, and $W$ toward the North Galactic Pole). The updated transformation matrix for J2000 coordinates was taken from the Hipparcos catalog introduction, and the correction to the local standard of rest (LSR) was done by removing the solar motion of Dehnen \& Binney (1998): $(U, V, W)_{\mathrm{Sun}}=(10.00,+5.25,+7.17) \pm(0.36,0.62,0.38) \mathrm{km} \mathrm{s}^{-1}$. A circular velocity of $V_{\text {circ }}=220 \mathrm{~km} \mathrm{~s}^{-1}$ was added to the derived velocities to convert from the heliocentric frame to Galactocentric frame velocities of $(U, V, W)_{\mathrm{GSR}}=(-86.7,158.5$, $-10.5) \pm(12.2,17.0,23.4) \mathrm{km} \mathrm{s}^{-1}$, where the uncertainties were derived from the errors in the three velocity measurements, again following the Johnson \& Soderblom method (see Table 2 for a summary of these kinematical quantities for ACS members in SA 76).

\section{ORBIT FROM ACS CANDIDATES: WHY IS THE MOTION NOT ALIGNED WITH THE VISIBLE STREAM?}

The orbit for the ACS candidates in SA 76 was integrated in the Johnston et al. (1995) Galactic potential, which consists of a Hernquist (1990) spheroidal bulge, a disk following the Miyamoto \& Nagai (1975) prescription and a logarithmic halo. 



Figure 11. Left panel: vector point diagram of all well-measured stars in SA 76 (black dots), with the ACS members overplotted as open diamonds. Right panel: proper motions for only the ACS members, with error bars reflecting individual formal uncertainties.

(A color version of this figure is available in the online journal.)

As for all calculations in this work, we adopt $R_{\odot}=8.5 \mathrm{kpc}$ and $V_{\text {circ }}=220 \mathrm{~km} \mathrm{~s}^{-1}$. Orbit derivation was started at the position of SA 76 with the measured $(U, V, W)_{\mathrm{GSR}}$ components and a distance of $10 \mathrm{kpc}$, and was integrated both forward and backward for $1 \mathrm{Gyr}$, in order to map out the entire path and kinematics of the stream orbit. The orbit is shown in Galactic Cartesian $(X, Y, Z)_{\mathrm{GC}}$ coordinates in Figure 13, with the large dot representing the assumed position of ACS stars in SA 76, the solid line the backward orbit integration, and the dotted line the forward integration. This orbit has peri- and apo-galactic radii of $R_{p}=12.3_{-2.2}^{+2.3} \mathrm{kpc}$ and $R_{a}=18.5_{-0.4}^{+1.2} \mathrm{kpc}$, and an eccentricity of $e \equiv\left(R_{a}-R_{p}\right) /\left(R_{a}+R_{p}\right)=0.20_{-0.05}^{+0.08}$, where errors have been calculated by comparing orbits with the maximum and minimum space velocities from uncertainties in $U, V$, and $W$. Taking our measured $\vec{R}=(X, Y, Z)$ and $\vec{V}_{\text {tot }}=(U, V, W)$, we can derive the angular momentum (per unit mass) for this mean motion, $\vec{L}=\vec{R} \times \vec{V}_{\text {tot }}$. This yields a $Z$-component of angular momentum per unit mass of $L_{Z} \sim-2960 \pm 330 \mathrm{kpc} \mathrm{km} \mathrm{s}^{-1}$, placing the SA 76 ACS debris on a prograde orbit $\left(L_{Z}>0 \equiv\right.$ retrograde, $L_{Z}<0 \equiv$ prograde). The inclination of the orbit is estimated here as $\cos i=-L_{Z} / L$, resulting in $i=16.2_{-1.4}^{+3.4} \mathrm{deg}$.

To convert to a Galactocentric rest frame, we must remove the contribution of the Sun's $220 \mathrm{~km} \mathrm{~s}^{-1}$ circular velocity- $\left(\mu_{\alpha}\right.$ $\left.\cos \delta, \mu_{\delta}\right)=(0.45,-4.20)$ mas $\mathrm{yr}^{-1}$ for a non-moving object at $10 \mathrm{kpc}$ along the SA 76 line of sight - to the measured PMs. By subtracting this from the mean measured PM of ACS candidates in SA 76, we derive a Galactocentric PM of $\left(\mu_{\alpha} \cos \delta, \mu_{\delta}\right)^{\prime}=(-1.65,3.42) \pm(0.34,0.36)$ mas $\mathrm{yr}^{-1}$. The ACS is a distinct stream running almost north-south (in celestial coordinates) on the sky, so it is curious that the PM we derive from stars selected to be members of this stream is not oriented along that the same $\mathrm{N}-\mathrm{S}$ direction. This is evident in Figure 14, which shows the best-fit orbit from Grillmair et al. (2008, solid line) overlying an image of the matched-filter star-count density similar to that of Grillmair (2006), but using SDSS DR7. The GCM08 orbit was constrained by positions and RVs in two fields (ACS-B and ACS-C, shown as the small and large filled cyan squares in the figure), as well as 30 positions along the visible portion of the stream. This orbit traverses the vertical (i.e., roughly constant right ascension) stream and passes through the overdensity between $\alpha \approx 132^{\circ}-137^{\circ}, \delta \approx$ $0^{\circ}-10^{\circ}$ known as the EBS (G06) on the subsequent orbital wrap. The large (cyan) arrow centered on the SA 76 position is a vector representing the magnitude and direction of the mean (Galactocentric) PMs measured in SA 76, with yellow arrows on either side representing the $3 \sigma$ uncertainty in the total space 




Figure 12. Upper panel: measured values of $[\mathrm{Fe} / \mathrm{H}]$ for spectroscopic targets observed in SA 76 (solid histogram). The scaled Besançon model prediction for stars of similar magnitude and color as our spectroscopic targets is shown as the dot-dashed line. The model reproduces the observed metallicity distribution quite well. Lower panel: as in the upper panel, but with the dashed histogram depicting only those stars in our final sample of ACS members. There is a hint of an excess of metal-poor $([\mathrm{Fe} / \mathrm{H}] \lesssim-1.3)$ stars in the overall ACS member sample compared to model predictions, but we have too few ACS members to definitively assess this possibility. The solid, filled histogram shows the nine stars with well-measured metallicity (i.e., $\mathrm{S} / \mathrm{N}>20$ ). These are concentrated more to the metal-poor end than the model distribution, with a median $[\mathrm{Fe} / \mathrm{H}]=$ -1.26 , but with a broad abundance spread as expected for, e.g., a dwarf galaxy stellar population.

motion. The motion is not aligned along the obvious stream (though it is consistent with being so at the $\sim 3 \sigma$ level), but traces a path roughly along the orientation from the EBS to SA 76. In this section, we will explore in detail some possible explanations for the misalignment of our PM and the visible path of the ACS.

\subsection{Comparison to Grillmair et al. (2008) Orbit for the ACS}

The orbit we have derived from kinematics of ACS candidates has peri- and apo-galactic radii of $R_{p}=12.3_{-2.2}^{+2.3} \mathrm{kpc}$ and $R_{a}=$ $18.5_{-0.4}^{+1.2} \mathrm{kpc}$, and an eccentricity of $e=0.20_{-0.05}^{+0.08}$. This is a slightly more elongated orbit than the one found by GCM08 for the ACS, which was found to have $e \sim 0.1$, but with a nearly identical apogalacticon $(18.5 \mathrm{kpc}$ versus $19.0 \pm 1.9 \mathrm{kpc}$ from GCM08). GCM08 ruled out a retrograde orbit for the ACS based on SDSS/USNO-B PMs; we further note that the retrograde model in that work predicted extremely large

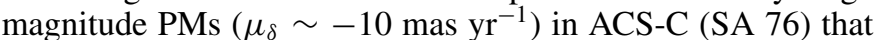
we can definitively rule out based on our current measurements. The inclination of our orbit for ACS candidates was found to be $i=16.2_{-1.4}^{+3.4} \mathrm{deg}$. This inclination angle is seemingly consistent with the result ( $i=20.1$ ) from GCM08; however, we note that the estimates derived in this way do not give the orientation of the orbital plane, but only the angle it makes to the $X Y$ plane. At the position of SA 76, we find $(U, V, W)_{\mathrm{GSR}}=(-52.4$, $207.4,79.0) \mathrm{km} \mathrm{s}^{-1}$ for the GCM08 orbit (based on kinematical quantities from their Table 2). The relative $Z$-components of the total velocity, $W_{\mathrm{GSR}}$, for this orbit and our PM-derived result are in opposite directions, indicating that the GCM08 orbit is oriented away from the Galactic disk at $i \sim 20^{\circ}$, while the PMs indicate movement downward toward the disk at $i \sim 20^{\circ}$ (this difference in orientation can be seen in Figure 14).

GCM08 found that a stream orbit constrained by 30 fiducial points along the spatial distribution and their measured RVs in two fields along the stream should produce PMs at the position of ACS-C (SA 76) of $\left(\mu_{\alpha} \cos \delta, \mu_{\delta}\right) \sim(0.64,0.67) \pm$ $(0.03,0.35)$ mas $^{-1} r^{-1}$ (for the model unconstrained by SDSS/ USNO-B PMs). This disagrees with our measurements by (1.84,

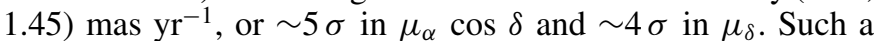
discrepancy cannot be due to the $\sim 0.43$ mas $\mathrm{yr}^{-1}$ uncertainty in our SA 76 PM zero point, which is robustly determined by $\gtrsim 100$ point-like galaxies in each field. An offset of this magnitude $\left(\Delta \mu \approx 2.4\right.$ mas $\mathrm{yr}^{-1}$ in total $\left.\mathrm{PM}\right)$ corresponds to a difference in tangential velocity of $>110 \mathrm{~km} \mathrm{~s}^{-1}$ at $10 \mathrm{kpc}$, so it is unlikely to be simply a measurement error. As will be discussed in Section 6.2, comparison of our measurements with the expected PMs along this line of sight from the Besançon model shows no obvious offset between the model-predicted motions of smooth Galactic populations and our results, so we do not believe a systematic PM error is present in our derivations. However, the total three-dimensional velocity we derive differs by only $\sim 44 \mathrm{~km} \mathrm{~s}^{-1}(\sim 1.5 \sigma$ using the uncertainty in our measured $U V W$ velocity) from the GCM08 orbit at the position of SA 76, and we will argue further in Section 6.5 that the two results are consistent with having originated from separate components of a substructured progenitor (e.g., a dwarf galaxy with associated globular clusters), but that in SA 76 we may not be measuring the motion of the main body of the stream.

\subsection{Is the "Misalignment" Due to Systematic Errors in the Proper Motions?}

SA 76 lies along the visible stream (though apparently on the periphery of the ACS rather than on a region of highest density; see Figures 14 and 18), and inspection of the CMD shows a clear overdensity of faint, blue stars in this field. Stream candidates were selected from our SA 76 PM database to be consistent with membership in a structure at $\sim 10 \mathrm{kpc}$ distance. We have shown (Section 3 ) that the expected velocity distribution of field stars (including both MW halo and thick disk) among our selection criteria is reproduced well, so that the significant number of ACS candidates in the narrow velocity peak suggests that the overdensity is real. The clear narrow peak among RVs of these candidates confirms that we are indeed sampling members of a comoving population among these candidates. Because the stream traces such a distinct swath across the sky, we expect the tangential motions of stars within the identified velocity peak in SA 76 to follow this stream. The fact that the three-dimensional space velocity of the ACS candidates is directed at a $\sim 30^{\circ}$ angle to the visible stream led us to explore the possibility that there was a systematic error in our PM measurements. 




Figure 13. Orbit based on the measured $(U, V, W)$ space velocity in SA 76 . The distance was taken to be $10 \mathrm{kpc}$ at the position of SA 76, and the orbit integrated both backward (solid lines) and forward (dotted curves) for $1 \mathrm{Gyr}$ (about 2.5 orbits) to trace out the full orbital path.

\subsubsection{Comparison to the Besançon Galaxy model}

Because we have reliably measured PMs for most $g \lesssim 20.5$ stars in the field of view, and many as faint as $g=21$, we chose to use stars well outside the ACS candidate selection regions to assess how well we recover the expected motions of Galactic stellar populations. To do this, we selected faint $(18<g<21)$, red $(1.1<g-r<1.7)$ stars from both the SA 76 PM catalog and the Besançon model predictions for the same line of sight. At these red colors, nearly all the faint stars thus selected should be nearby (foreground) MW M dwarfs (and contain no ACS debris). Figure 15 shows the equatorial PMs in each dimension for these $\mathrm{M}$ dwarf candidates-the upper panels depict measurements in SA 76, and the lower panels the model predictions. Gaussian fits to these distributions (dashed lines in the figure) have peaks at $\left(\mu_{\alpha} \cos \delta, \mu_{\delta}\right)_{\mathrm{SA} 76}=(-2.42,-2.86)$


$-2.53) \pm(0.16,0.17)$ mas $\mathrm{yr}^{-1}$. These agree at the $\sim 1 \sigma$ level and differ by less than the $\sim 0.4$ mas $\mathrm{yr}^{-1}$ uncertainty in our PM zero point. We further examined our PMs for residual colorand magnitude-dependent systematics, and found no significant trends (as evidenced by the lack of slope in the mean PMs with either magnitude or color in Figure 9).

To investigate whether contaminant MW thick disk and halo stars are skewing our mean PMs for ACS candidates, we selected all stars from the Besançon model within the same color and magnitude criteria as our ACS candidates $(18.5<g<20.5$, $0.2<g-r<0.6$ ). Gaussian fits to the PMs of these selected model populations yield $\left(\mu_{\alpha} \cos \delta, \mu_{\delta}\right)_{\text {model }}=(-0.36,-2.66)$ $\pm(0.06,0.09)$ mas $\mathrm{yr}^{-1}$. This differs by $\sim(2.5,5.2)-\sigma$ from our mean ACS PMs of $\left(\mu_{\alpha} \cos \delta, \mu_{\delta}\right)_{\mathrm{SA} 76}=(-1.20,-0.78) \pm$


any expected due to uncertainties in the PM zero point, and, as shown in the previous paragraph, are unlikely to result from a systematic offset in the PMs. Thus, any non-stream members (i.e., thick disk or halo stars) that have been misidentified as ACS candidates have likely skewed the PM measurements to higher values in $\mu_{\alpha} \cos \delta$, and shifted our estimate of $\mu_{\delta}$ lower than its intrinsic value. The component of PM along right ascension one would measure for an object at a distance of $10 \mathrm{kpc}$ moving along the visible stream (i.e., with motion along constant right

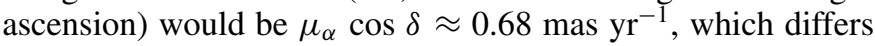
by $\sim 5.5 \sigma$ from our measured value for ACS candidates. Our measurement for candidate ACS debris stars is lower than both this prediction and the expected PMs of MW populations along this line of sight. Thus, the misalignment of our measured PM from the expected stream motion cannot be due to contamination of our sample by foreground MW stars, which would actually bring our measurements closer to the expected stream motion. In addition, the magnitude of the Besançon-predicted total PM, $\mu_{\text {model }}=2.68$ mas $\mathrm{yr}^{-1}$, is nearly twice that of the PM we measured $\left(\mu_{\text {SA76 }}=1.43\right.$ mas yr $\left.^{-1}\right)$ for ACS debris-a difference of $\sim 3.5 \sigma$. The difference between our mean PMs and the 



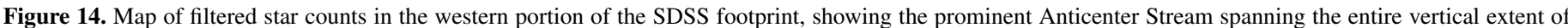

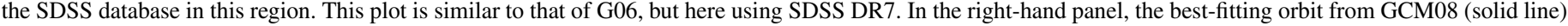

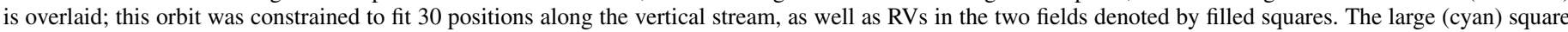

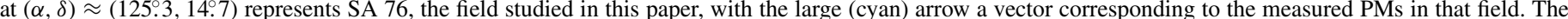





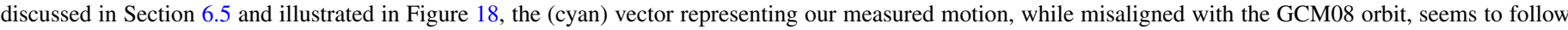

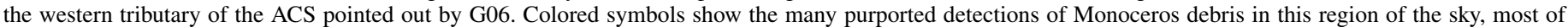
which are not spatially coincident with the ACS.

(A color version of this figure is available in the online journal.)

expected stream and foreground star kinematics is significantly larger than can be accounted for by the uncertainties in the PM zero point, which are at the $\sim 0.4$ mas $\mathrm{yr}^{-1}$ level in each PM dimension. Based on all of the above arguments, we conclude that the difference between the orientation of the three-dimensional space motion we measure for purported ACS debris and the expected direction of motion is not due to systematic errors in the PMs, but is a real kinematical difference.

\subsubsection{Direct Comparison to SDSS/USNO-B Proper Motions}

Another means of determining whether systematic offsets are present in our data is to compare individual PM measurements between our catalog and another from the literature. The SDSS/USNO-B PMs (Munn et al. 2008) were chosen for this comparison because (1) SA 76 is within the SDSS footprint, and should thus have SDSS/USNO-B PMs available for most objects in the field and (2) the SDSS/USNO-B PMs were used by GCM08 to constrain the orbit of SA 76 using their smaller sample of RV members. To look for systematics in the zero point used to fix the absolute reference frame, individual absolute PMs were compared for all objects in common between the catalogs that were identified as galaxies in SDSS (upper panels of Figure 16). The two upper panels show our measurements on the left and the SDSS/USNO-B PMs on the right, with the size of a typical uncertainty given by the error bar in the upper left corner of each plot. Because the SDSS/USNO-B PMs are given by Munn et al. (2008) to integer precision, many of the points will overlap each other in such a plot. To make it easier to differentiate points in the figure, we


dimension to the SDSS PMs. Our measurements are more tightly clumped than the SDSS data, suggesting that our results are more reliable for these distant, zero-PM galaxies. The mean PMs from these distributions (clipped at 2.5 $)$ are $<\left(\mu_{\alpha} \cos \right.$ $\left.\delta, \mu_{\delta}\right)>_{\text {SA76 }}=(-0.12,-0.30) \pm(0.39,0.33){\text { mas } \mathrm{yr}^{-1} \text { and }<}$ $\left(\mu_{\alpha} \cos \delta, \mu_{\delta}\right)>_{\text {SDSS }}=(0.09,0.66) \pm(0.55,0.47)$ mas $\mathrm{yr}^{-1}$. The mean SDSS/USNO-B PM of galaxies is inconsistent with zero $\mathrm{PM}$ by $1.4 \sigma$ in $\mu_{\delta}$, while our measurements are consistent with zero in both dimensions. The galaxies' mean $\mu_{\alpha} \cos \delta$ from the two surveys agree within the uncertainties, suggesting that the difference between our measured $\mu_{\alpha} \cos \delta$ for ACS debris and the expected motion is not due to a zero-point uncertainty. Note, however, that the SDSS value of $\mu_{\delta}$ for galaxies differs by more 


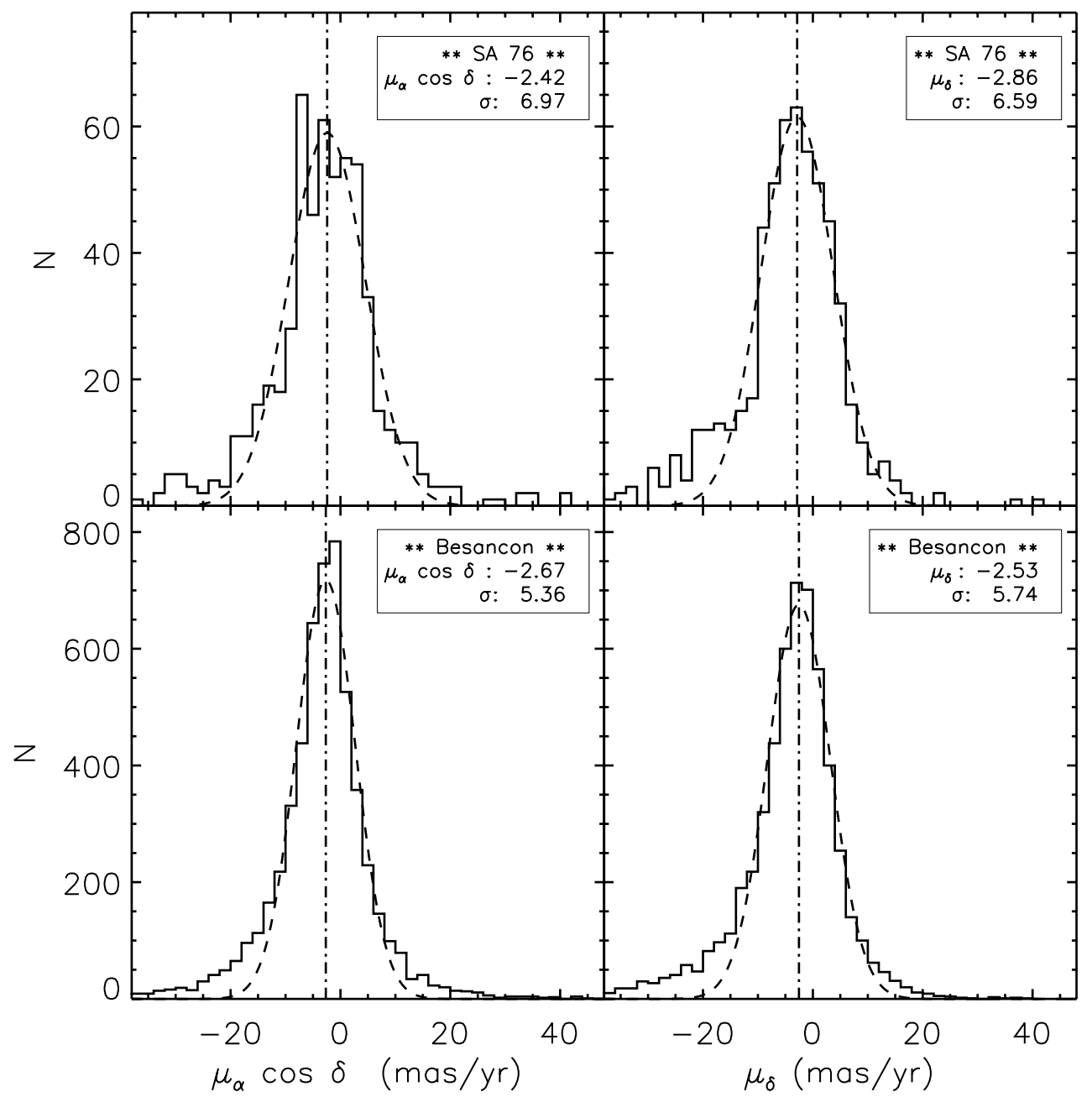

Figure 15. Proper motions in each dimension for faint $(18<g<21)$, red $(1.1<g-r<1.7)$ stars (selected to be nearby M dwarfs) in SA 76 (upper) and Besançon (lower). The agreement between the fitted mean values for $\mu_{\alpha} \cos \delta$ and $\mu_{\delta}$ for the two samples leads us to conclude that no large systematic error is present in our measured proper motions.

than $2 \sigma$ from our determination; because the precision of our measurements (as reflected by the individual PM uncertainties as well as the errors in the sigma-clipped means) is much better than that given by SDSS, we choose to retain our zero point rather than shift to the SDSS frame.

The lower panels of Figure 16 are similar to the upper panels, but compare PMs for faint $(g>17)$, blue $(0.0<$ $g-r<0.8)$ stars (excluding galaxies) in common between our SA 76 catalog and SDSS. The distributions are similar, but our PMs have roughly twice the precision of the SDSS/USNO-B determinations. This becomes even clearer when comparing only the final sample of 31 stars we have selected as ACS candidates (Figure 17). From these 31 stars, our data yield a PM with $\sim 0.35$ mas $\mathrm{yr}^{-1}$ precision in each dimension; the SDSS



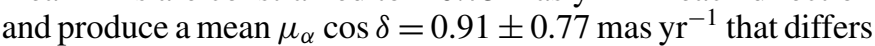
by $\sim 6 \sigma$ from our determination. Clearly the SDSS/USNO-B PMs do not have the requisite precision at faint magnitudes to constrain distant tidal stream kinematics.

\subsection{Is Sagittarius Tidal Debris Present among the ACS Candidates?}

Another stellar population that may be present along the SA 76 line of sight is leading tidal debris from the Sagittarius (Sgr) dSph. The Sgr stream is prominently visible $\sim 5^{\circ}$ from the position of SA 76 in Figure 2 of GCM08 and our Figure 14. To determine the expected properties of Sgr debris in this region, we turn to the recent comprehensive model of the Sgr tidal debris system from Law \& Majewski (2010). Figure 9 of this work shows the expected positions, RVs, and distances for the Sgr stream in the SDSS footprint. SA 76, at $(\alpha, \delta)_{2000}=$ $\left(125^{\circ} .3,14.7\right)$, is on the periphery of the Sgr leading debris tail. All stars within $\pm 5^{\circ}$ (in both $\alpha$ and $\delta$ ) of the position of SA 76 were selected from the best-fit model of Law \& Majewski (2010), and their kinematical properties compared to stars in SA 76. The median expected RV for Sgr leading arm debris thus selected is $V_{\text {helio,Sgr }}=-47 \mathrm{~km} \mathrm{~s}^{-1}\left(V_{\mathrm{GSR}, \mathrm{Sgr}}=\right.$ $-143 \mathrm{~km} \mathrm{~s}^{-1}$ ), with a dispersion of $\sim 15 \mathrm{~km} \mathrm{~s}^{-1}$. This is well outside the ACS candidate velocity selection we have used, so we expect no contamination from Sagittarius debris in our ACS sample (note also that there is no clear excess at the Sgr debris velocity in Figure 6, so we do not seem to be sampling much, if any, Sgr leading debris in SA 76). In addition, Law \& Majewski (2010) predict Sagittarius leading arm debris to be at a distance of $\sim 15-20 \mathrm{kpc}$ in this region, which would place the MSTO of Sgr debris $\sim 0.5-1.0$ mag fainter than the MSTO seen in SA 76.

\subsection{Is the EBS Associated with the Anticenter Stream?}

The ACS orbit fit by GCM08 passes through the overdensity at $(\alpha, \delta)_{2000} \sim\left(134^{\circ}, 3^{\circ}\right)$, dubbed the Eastern Banded Structure 



Figure 16. Upper panels: proper motions of all objects identified as galaxies by SDSS that appear in our SA 76 data set and have SDSS/USNO-B proper motions in the catalog derived by Munn et al. (2008). The left panel shows our measurements, and the right panel the SDSS PMs; in each plot, a representative error bar depicts the mean uncertainty for the PM measurements. The SDSS/USNO-B proper motions are given to integer precision in the catalog of Munn et al. (2008); for plotting purposes only, we have applied random shifts of between $\pm 0.5 \mathrm{mas} \mathrm{yr}^{-1}$ in each dimension before plotting the points, in order to differentiate overlapping points. Sigma-clipped (at $2.5 \sigma)$ mean PMs from these distributions give $<\left(\mu_{\alpha} \cos \delta, \mu_{\delta}\right)>_{\text {SA76 }}=(-0.12,-0.30) \pm(0.39,0.33) \mathrm{mas} \mathrm{yr}^{-1}$ and $_{\delta}<\left(\mu_{\alpha} \cos \right.$ $\left.\delta, \mu_{\delta}\right)>$ SDSS $=(0.09,0.66) \pm(0.55,0.47)$ mas $\mathrm{yr}^{-1}$. These determinations are consistent in $\mu_{\alpha} \cos \delta$ within the uncertainties, suggesting that no global offsets are present in that dimension of the proper motions. However, there is a $\sim 2 \sigma$ offset in $\mu_{\delta}$; because the precision of our measurement is superior to that of the SDSS determination, we choose to retain our proper motion zero point rather than offsetting to the SDSS/USNO-B frame. Lower panels: as in the upper panels, but showing proper motions of all faint $(g>17)$, blue $(0.0<g-r<0.8)$ stars (excluding galaxies) in common between our SA 76 catalog and SDSS. The distributions appear similar, with less scatter in our more precise measurements compared to the SDSS data.

(or EBS) by G06, on a subsequent orbital wrap, leading GCM08 to suggest that the ACS and EBS may be associated. In GCM08 and G06, a large, $\sim 5^{\circ}$ gap in the coverage of SDSS DR5 passed between the EBS and the ACS. Since the publication of GCM08, SDSS Data Release 7 (DR7) has been made public. We have used this more complete photometric catalog to generate new filtered star-count maps of the Sloan footprint (Figure 14). With the gap now filled in DR7, the EBS overdensity does not appear to continue west toward the main ACS. This may be because the stream associated with the EBS is curving or inclined away from us, and there are simply fewer stars contributing to the star counts at fainter magnitudes. It could also be that the portion of the stream to the west of the EBS is "clumpy," and there is little debris between SA 76 and the main EBS feature. Of course, the overdensity at $\sim\left(134^{\circ}, 3^{\circ}\right)$ may simply be where the EBS stops. The orbit derived from the kinematics we measured in SA 76, if traced backward from SA 76, would pass through (or near) the EBS, but the discontinuity between SA 76 and the EBS "blob" makes it seem unlikely that the EBS is debris at small angular separation along the same stream as that sampled by our candidates in SA 76. However, this does not rule out an association between the EBS and the whole of the ACS-a larger-scale, deep kinematical study would be necessary to assess their possible physical association.

\subsection{Are We Measuring the Motion of a Tributary or Substream of the Main Anticenter Stream?}

Grillmair (2006) found that the overdensities of star counts in the ACS separate into three distinct components: a broad $\sim 2^{\circ}$ wide stream running down the center of the system, with two (or more) narrower streams on the east and west side of the broad 


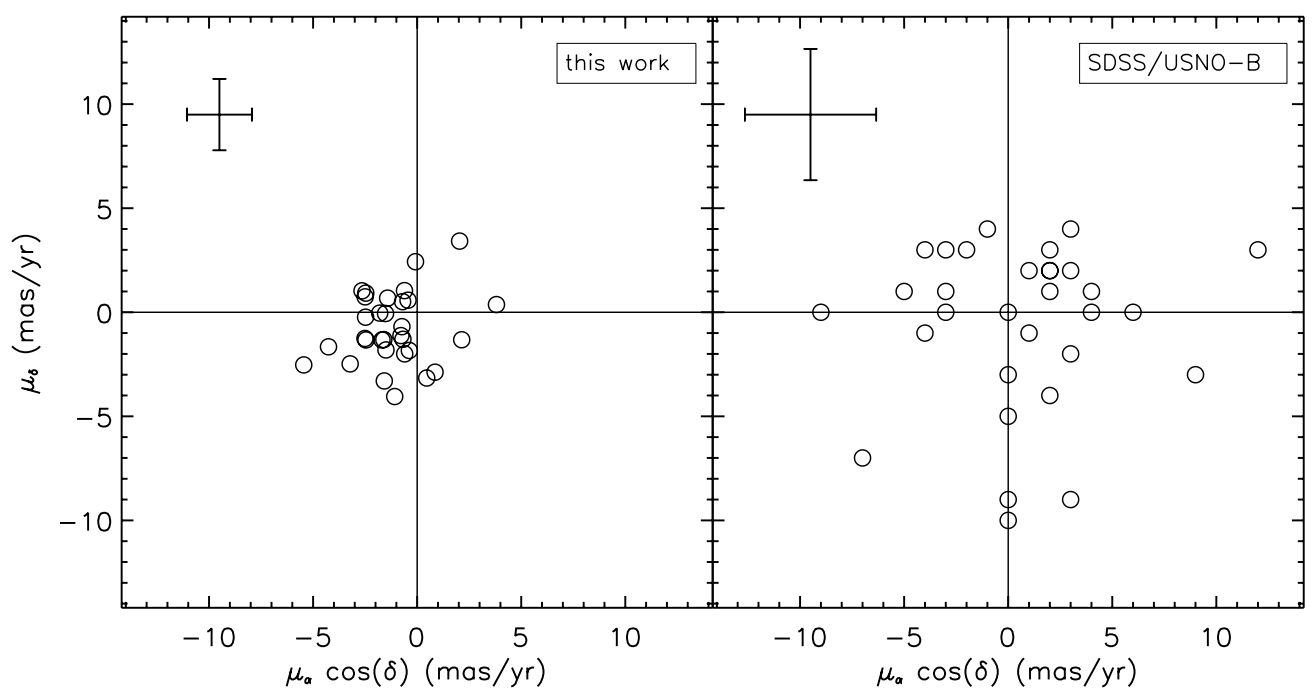

Figure 17. Proper motions of the final sample of 31 ACS candidates. The left panel shows our measurements; the right panel those from SDSS/USNO-B. Error bars in each panel represent the typical PM uncertainty. The SDSS proper motions are clearly inconsistent with our measurement for ACS debris: $<\left(\mu_{\alpha}\right.$ cos

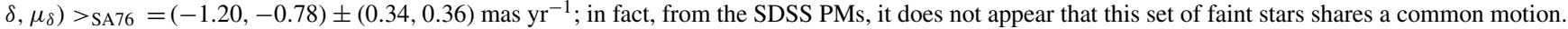

component. Their Figure 2 shows total star counts as a function of right ascension in slices at constant declination across the stream. It is clear from that figure that there are at least three main components and probably a number of other sub-streams making up the ACS complex. Thus, in order to measure the bulk motion and derive an orbit for the stream progenitor, it may be necessary to disentangle these tributaries and assess the contributions of residual motion about a common center of mass (i.e., the progenitor dwarf galaxy) that the separate components may have shared.

The measured motion of ACS candidates in SA 76 (represented as a vector by the cyan arrow in Figure 14) appears to follow the western substream studied by G06, which loops outward to the west and separates from the main (or "central") stream component to the north of SA 76 in Figure 14. The orientation of our derived ACS motion from SA 76 stars may represent the peculiar motion of this substream about the orbital center of the stream progenitor. Figure 18 depicts the same star-count map, but zoomed to the region near SA 76. In this figure, we have traced a possible course of the western substream, which is guided in part by the peaks identified by G06 in profiles of star counts taken across the stream at positions of constant declination. This is simply shown as a guide to the reader to illustrate our suggestion that the SA 76 kinematics may reflect the motion of the western tributary. Note also that the open box denoting the position of SA 76 in Figure 18 is in a region of lower stream density (white in the figure) on the periphery of the ACS.

As mentioned previously, the total three-dimensional space velocity we measured $\left(V_{\mathrm{tot}} \approx 181 \mathrm{~km} \mathrm{~s}^{-1}\right)$ differs by only $44 \mathrm{~km} \mathrm{~s}^{-1}$ from the best-fit ACS model motion of GCM08 at the position of SA $76\left(V_{\text {tot }} \approx 225 \mathrm{~km} \mathrm{~s}^{-1}\right)$. In testing the possibility that Pal 12 was previously associated with the Sagittarius dSph, Dinescu et al. (2000) calculated an escape velocity of $\sim 40 \mathrm{~km} \mathrm{~s}^{-1}$ for a globular cluster bound to an Sgrlike $10^{8} M_{\odot}$ satellite. Thus, the relative velocities are consistent with components such as globular clusters or other distinct kinematical overdensities within the progenitor. Furthermore, the angle between these three-dimensional velocity vectors is only $\sim 27^{\circ}$. This fact taken together with the similarity of the space velocities suggests that the orbits, though oriented in slightly different directions, may be associated, with their slight misalignment a remnant of peculiar motions about the center of mass of the progenitor system. Moreover, the integrals of motion for both orbits are similar: the total angular momenta (per unit mass) for the two orbits agree within $\sim 20 \%-25 \%$, and their orbital energies differ by $\sim 10 \%-15 \%$. This is suggestive of similar orbits for the main ACS stream and the motion we have measured in SA 76, in spite of the misalignment with the visible stream. It may be that the ACS represents the remnant of a progenitor satellite that originally had its own satellites orbiting within its gravitational potential, similar to the globular clusters associated with the Sgr dSph (e.g., Da Costa \& Armandroff 1995; Dinescu et al. 2000; Bellazzini et al. 2003) or the Fornax dSph (see, e.g., Forbes et al. 2000). It is also possible that this richly substructured stream complex is the result of an infalling group of associated satellites that fell into the MW together, as in, e.g., Li \& Helmi (2008) or D'Onghia \& Lake (2009).

The low-inclination, nearly circular orbit derived for the ACS suggests that one may expect debris from multiple wraps of the best-fit orbit (or two separate sub-systems) to be present in SA 76, perhaps with distinct RV signatures. Debris from the subsequent wrap of the orbit in GCM08 passes through the ACS locus at a distance of $\sim 15 \mathrm{kpc}$ (though at a declination $10^{\circ}-20^{\circ}$ north of SA 76). We will consider this as a reasonable distance for debris from a second orbital wrap. A difference of 3-5 kpc in distance corresponds to an offset of $\Delta g \sim 0.6-1.0 \mathrm{mag}$ in the CMD. It is unlikely that these two overlapping stellar populations can be separated (while also separating stream members from Galactic contamination) without accurate RVs from medium-to-high resolution spectra for all stars at the faint end of our survey. There is the hint of a concentration of stars blueward of the obvious ACS main sequence, but below the ACS turnoff (at $g \sim 21, g-r \sim 0.3$ ) in the CMD of Figure 10 (left panel), suggestive of a second MSTO. However, this occurs near the magnitude limit of the PM survey, and we are unable to explore the possibility of a second population with the data in hand. We do note that the apparent scatter in PMs for bright $(g<18.5)$ stars (candidate ACS red giants) discussed in Section 3 could arise from the superposition of multiple RGBs. If this is the case, it would also explain the lack of a narrowly defined RGB among brighter stars with ACS-like 


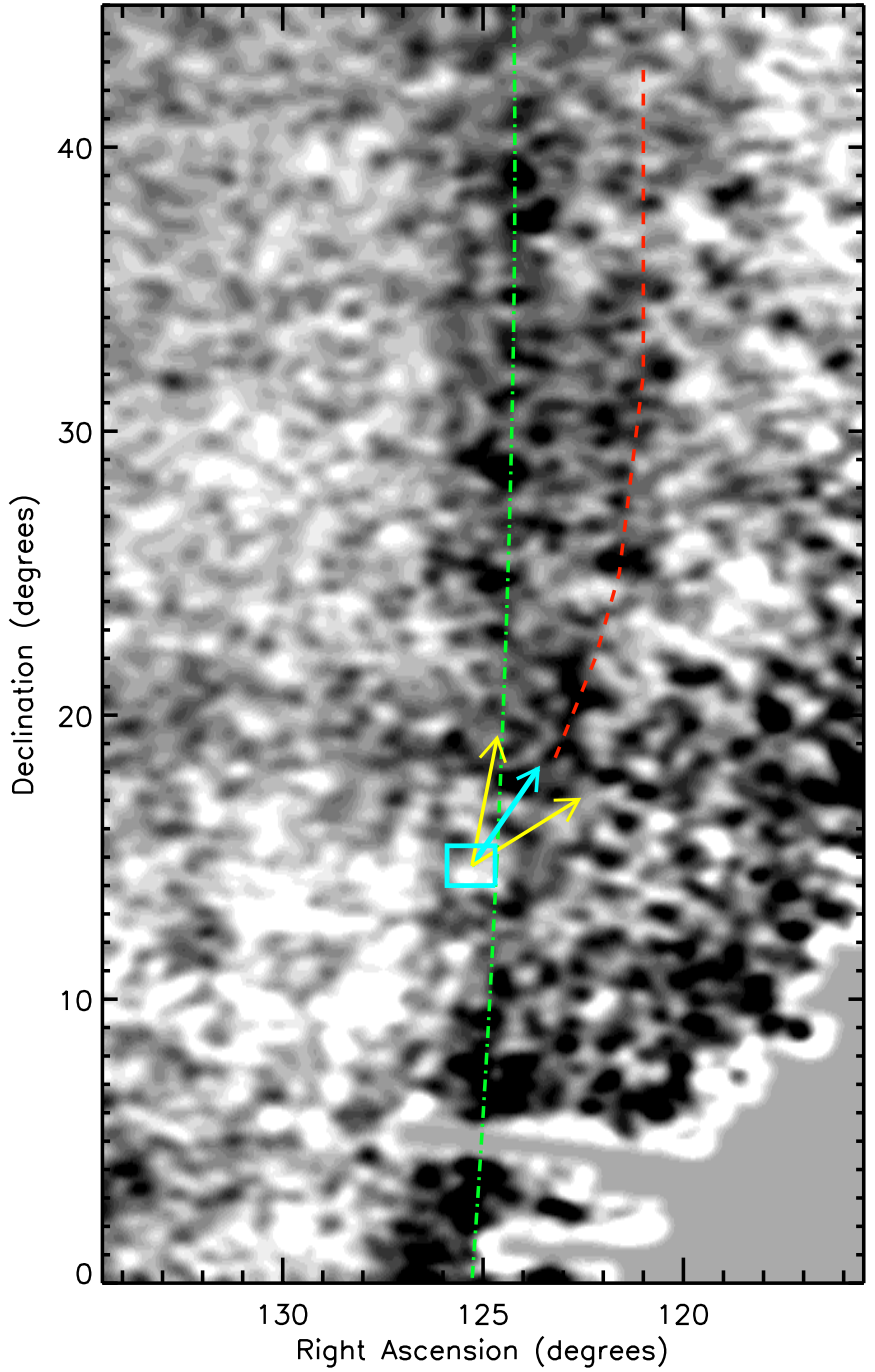

Figure 18. Star-count map (as in Figure 14) with the orbit of GCM08 shown by the (green) dot-dashed line, and SA 76 denoted by the open square. A path of the Western substream possibly traced by ACS debris with kinematics as measured in SA 76 is highlighted as a (red) dashed curve. This curve is loosely based on the peaks identified as the "West" substream by G06, as well as visual examination of the star-count map and extrapolation of the proper motion vector. (A color version of this figure is available in the online journal.)

RVs, suggesting that the large PM scatter in RGB-like stars is a reflection of true differences in PMs of different populations. The presence of multiple wraps of stellar debris in SA 76 could also explain the large metallicity spread seen in Figure 12. Many dSphs are known to have radial gradients in $[\mathrm{Fe} / \mathrm{H}]$, and, as shown by Chou et al. (2007) for the Sagittarius tidal tails, the preferential stripping of outer populations from dSphs gives rise to metallicity gradients along the debris streams. If the ACS arises from such a scenario, the superposition of two populations from multiple orbital wraps (thus having different mean metallicities) in SA 76 could then produce a broader metallicity distribution than expected from a single population.

\subsection{Conclusion: The Origin of the Unexpected Kinematics in SA 76}

Though the reason our measured kinematics in SA 76 are not oriented along the visible ACS remains unclear, we have ruled out systematic PM errors and Sagittarius tidal debris as the origin of the misalignment. The space velocity we have measured for ACS candidates in SA 76 is similar to that of the GCM08 orbital fit, is directed along a visible substream, and has motion relative to the main stream similar to what is seen for globular clusters that orbit other Local Group dwarf galaxies (either intact or disrupted). Based on these properties, we argue that our measurements reflect the motion of a tributary of the main ACS system. This substream may be the remnant of either a globular cluster or companion dwarf galaxy that was bound to the larger system that was the ACS progenitor.

\section{ARE THE ACS AND MONOCEROS RELATED?}

There have been numerous studies of stellar systems in the anticenter and outer Galactic disk region all purporting to be Monoceros (also known as "GASS") debris (Newberg et al. 2002; Crane et al. 2003; Ibata et al. 2003; Yanny et al. 2003; Wilhelm et al. 2005; Vivas \& Zinn 2006; Conn et al. 2007). In this section, we attempt to sort out the many detections and determine (based on our measured kinematics) whether the distinct ACS feature is related to Mon. The many purported Mon detections within the western SDSS footprint are highlighted as various colored symbols in the star-count map of Figure 14. From this figure, it is clear that Mon, as reported in previous work, would be a large feature spanning much of the low-latitude sky near the Galactic anticenter. The ACS, on the other hand, was eventually revealed to be a narrow, well-defined stellar stream (with even narrower substreams) by G06. A few of the claimed Mon detections appear to be spatially coincident with the ACS (e.g., the points from Wilhelm et al. 2005, one of the Newberg et al. 2002 fields, the Ibata et al. 2003 point, and a handful of the $\mathrm{M}$ giants from Crane et al. 2003), while the majority of the remaining Mon candidates are at lower latitudes than the ACS. It is possible that the two systems are associated, but if they are distinct systems, some of the stars and overdensities previously associated with Mon may actually be members of the ACS. Accurate kinematical and stellar abundance study of large numbers of stars in this region (preferably covering a contiguous area) may make it possible to determine whether these are associated features of the same disruption event, a spatial coincidence of unrelated systems, or confusion of not well discriminated early studies of structures near the Galactic antienter. In this section, we compare the properties of Monoceros and the ACS to explore their possible association.

\subsection{Metallicities}

The abundance spread for claimed Monoceros detections is not fully understood, with a variety of conflicting results making this feature difficult to understand. One of the earliest studies of Monoceros (Yanny et al. 2003) estimated [Fe/H] $=-1.6 \pm 0.3$ from SDSS spectra of MSTO F stars, while Crane et al. (2003) found a much higher $[\mathrm{Fe} / \mathrm{H}]=-0.4 \pm$ 0.3 for Two Micron All Sky Survey selected M giants. From these two results alone, it is obvious that if the various Mon overdensities derive from a single structure, then there must have been a significant metallicity spread within the Mon progenitor. Ivezić et al. (2008) derive $[\mathrm{Fe} / \mathrm{H}]=-0.95$ for Monoceros based on photometric metallicites of thousands of Fand G-type dwarfs near the Galactic anticenter from the SDSS database, and also show that this metallicity is quite distinct from the MW halo and disk in this direction (see their Figure 18). Our result for the ACS is generally rather similar to these results for Monoceros, in that we find a predominantly metal- 
poor population, but with broad scatter. Additional work on A/F-type stars by Wilhelm et al. (2005) found $<[\mathrm{Fe} / \mathrm{H}]>=$ -1.37 , with scatter of $\sim 0.5$ dex - both their mean metallicity and the spread of measurements are comparable to what we have found for the ACS from stars of similar spectral type. The stars in this Wilhelm et al. (2005) study are located in two SDSS plates that are on or near the obvious ACS feature in Figure 14 (where Wilhelm et al. 2005 fields are shown as open red circles). The population identified as Mon debris in the southernmost of these fields (Plates $1149 / 1154$, at $(\alpha, \delta) \sim(125.1,2.7))$ had measured RV of $88 \mathrm{~km} \mathrm{~s}^{-1}$, which is consistent with the RV for the ACS orbit derived by GCM08 at that position. Thus, though Wilhelm et al. (2005) have claimed these to be Monoceros stars, it is likely that their study is sampling ACS debris from the same populations we are studying in SA 76.

Finally, we note that Chou et al. (2010) show that many M giants (selected from Crane et al. 2003) in their high-resolution spectroscopic study near the anticenter have $[\mathrm{Fe} / \mathrm{H}] \sim-0.9$ (as expected from Ivezić et al. 2008), with a tail extending to higher metallicities (confirming the results of Crane et al. 2003). This work shows that these Mon M giants have $\alpha$ and $s$-process elemental abundance trends similar to those of $\mathrm{M}$ giants in the Sagittarius tidal tails, as well as other dSphs. While this suggests a dwarf galaxy origin for the Monoceros stars, it cannot definitively rule out that Mon is the result of a "puffing up" of the disk due to an encounter, since the outer disk itself may be formed hierarchically from merger events. The similarity of the rather narrow ACS (which is likely the result of a dSph disruption) to the much more broadly distributed Monoceros feature could be because the ACS is the coherent remnant of a larger disruption event that produced the entire Mon "ring." Alternatively, it may simply be that spatial coincidence has led to the conflation of two distinct structures and that confusion leads to ACS members "contaminating" determinations of Mon properties.

\subsection{Kinematics}

Predicted Galactocentric RVs as a function of Galactic longitude are shown in Figure 19 for our derived orbit of SA 76 ACS debris (top panel), with the best-fit orbits from GCM08 (ACS) and Peñarrubia et al. (2005, Mon) in the lower two panels. A similar plot in Figure 20 shows the spatial distribution of the ACS and Monoceros orbits in Galactic coordinates. Comparison data from the literature are shown in both figures using the same colors and symbols as in Figure 14 for claimed Monoceros detections near our SA 76 field. The large filled square representing our measured velocity for ACS debris at the position of SA 76 is slightly outside the hatched region in Figure 19, which represents an object in a circular orbit with a velocity of $220 \mathrm{~km} \mathrm{~s}^{-1}$ at $R_{\mathrm{GC}}=18 \mathrm{kpc}$, with a dispersion of $30 \mathrm{~km} \mathrm{~s}^{-1}$ (similar to Crane et al. 2003; note that, assuming a flat MW rotation curve, this velocity and dispersion are thin disk like). Our newly derived orbit is the only one of the three shown that exhibits significantly non-circular motion (i.e., extends well outside the hatched region) in Figure 19. The orbit derived by Peñarrubia et al. (2005) for Monoceros predicts a velocity in SA 76 that is similar to what we have measured, but cannot reproduce the spatial distribution of the ACS, as noted in GCM08. We note, however, that our orbit derived from kinematics in SA 76 also fails to reproduce the swath of ACS debris (the grey "bar" in Figure 20) that spans the entire declination range of the western SDSS footprint. Our derived orbit traverses slightly higher Galactic latitudes in Figure 20

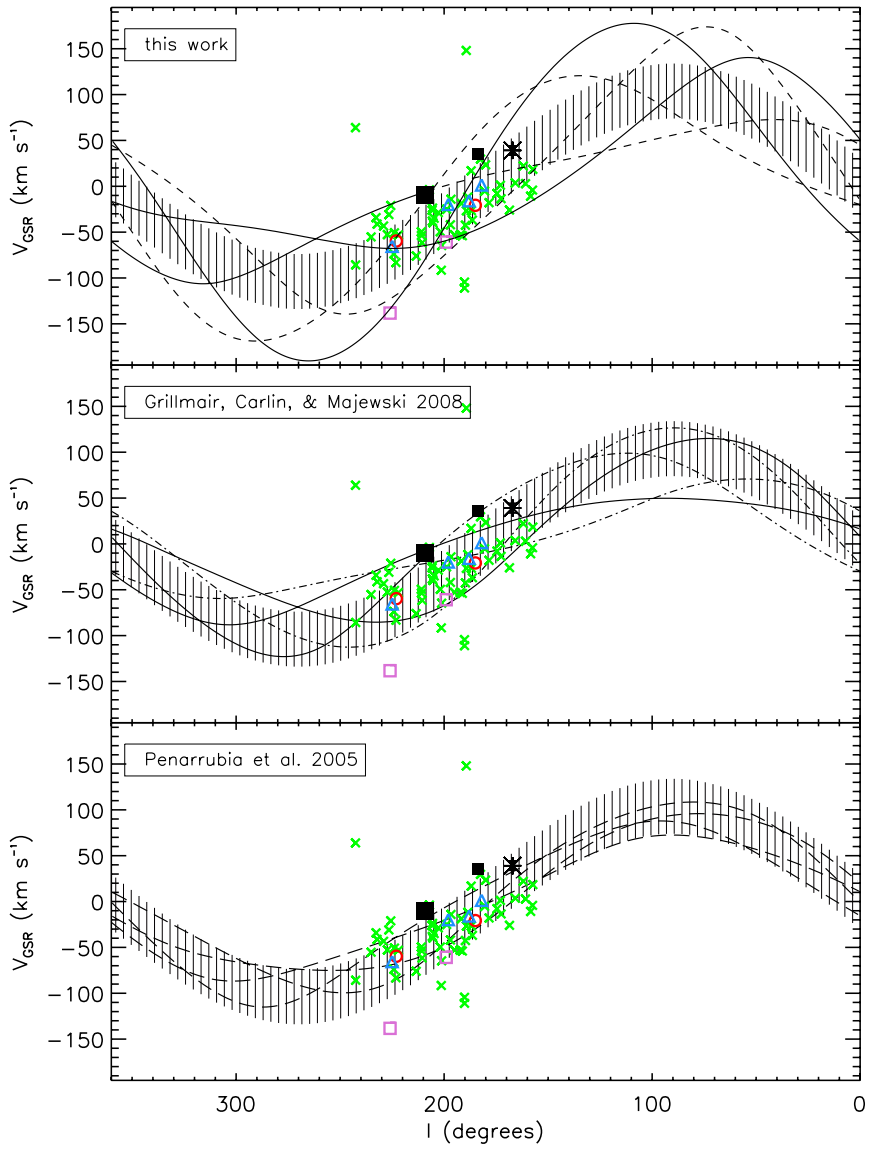

Figure 19. Galactocentric radial velocities as a function of Galactic longitude for our derived orbit (top panel; solid line: orbit integrated backward from SA 76 for $\sim 1$ Gyr, dashed line: forward integration), the Grillmair et al. (2008) orbit (middle panel; solid line: backward integration, dot dash: forward), and Peñarrubia et al. (2005) orbit (bottom; long dash). In each panel, a hatched region centered on the circular velocity curve is shown, as derived by Crane et al. (2003) to fit their velocities of candidate Monoceros M giants: $V_{\text {circ }}=220 \mathrm{~km} \mathrm{~s}^{-1}$ at Galactocentric distance of $R_{\mathrm{GC}}=18 \mathrm{kpc}$, with velocity dispersion of $30 \mathrm{~km} \mathrm{~s}^{-1}$. Also shown in each panel are Monoceros detections from the literature (symbols and colors as in Figure 14). The large asterisk shows the mean radial velocity for purported Monoceros debris in SA 71, a field in which Casetti-Dinescu et al. (2008) also measured three-dimensional kinematics. The error bar for this point is $\pm 5 \mathrm{~km} \mathrm{~s}^{-1}$, which is smaller than the size of the plotted point. A large filled square shows our measured velocity for ACS debris at the position of SA 76 and the smaller filled square represents the velocity of ACS debris in ACS-B (GCM08)

(A color version of this figure is available in the online journal.)

than the other two models, but is qualitatively quite similar to the result of GCM08, which suggests that our current study is measuring distinct "tributaries" or successive orbital wraps of the same stream system modeled in GCM08. The GCM08 orbit also reproduces many of the known detections of Mon debris in both velocity and position, but further detailed study incorporating all ACS and Mon data would be necessary to verify an association of the ACS with the Monoceros ring.

With three-dimensional kinematics, we can assess the similarity of the orbits derived by various studies by examining the integrals of motion. As discussed in Section 6, the mean motion we have measured in SA 76 corresponds to an angular momentum (per unit mass) of $L_{Z} \sim-2960 \pm 330 \mathrm{kpc} \mathrm{km} \mathrm{s}{ }^{-1}$, placing the SA 76 ACS debris on a prograde orbit. This differs by only $\sim 20 \%$ from the $L_{Z} \sim-3600 \mathrm{kpc} \mathrm{km} \mathrm{s}^{-1}$ predicted by the GCM08 ACS orbit along the SA 76 line of sight. Casetti-Dinescu et al. (2008) measured three-dimensional stellar 


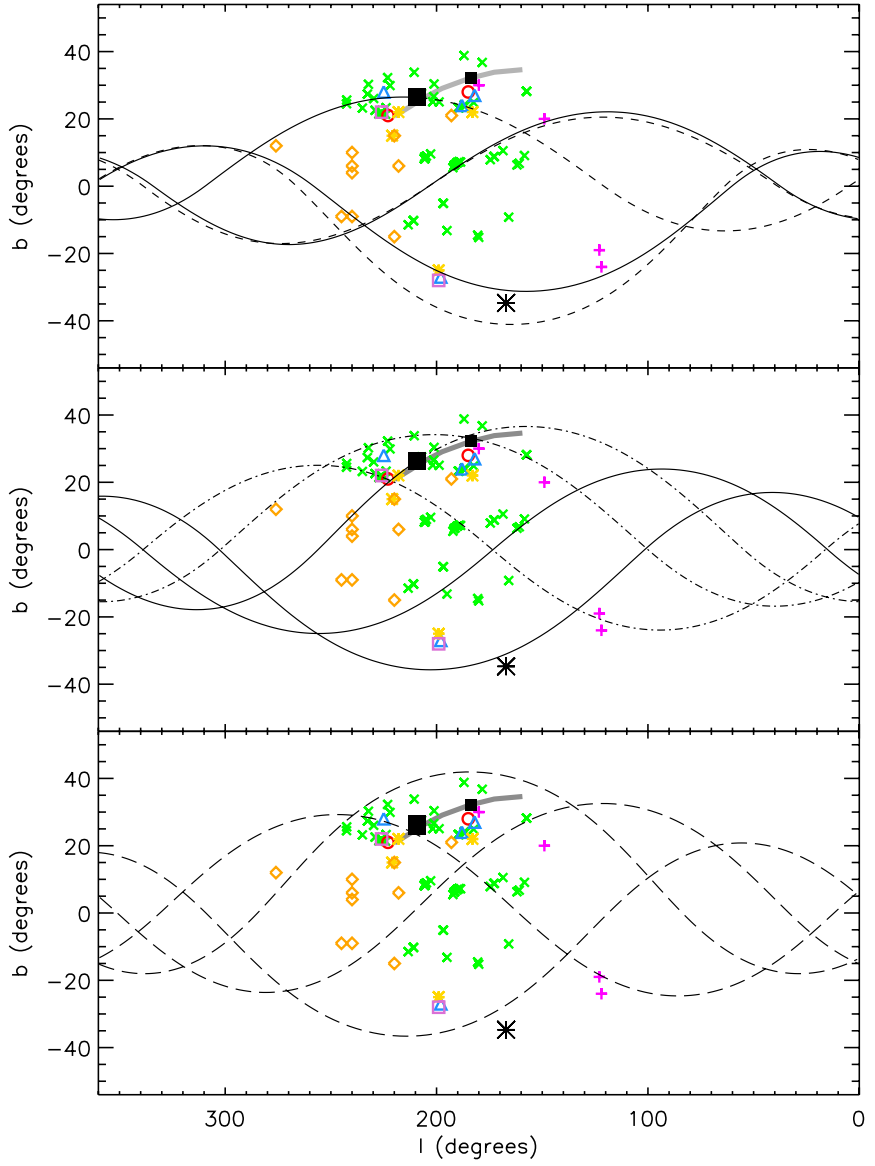

Figure 20. Orbital paths in Galactic coordinates, with panels and symbols as in Figure 19. The gray filled "bars" in each panel represent the area covered by ACS debris in the western portion of the SDSS footprint.

(A color version of this figure is available in the online journal.)

kinematics from an overdensity of purported Monoceros debris in SA 71 and found $L_{Z} \sim-3140 \pm 460 \mathrm{kpc} \mathrm{km} \mathrm{s}^{-1}$, which agrees with the ACS results within the uncertainties. The orbit derived from our kinematics in SA 76 produces a RV near the Casetti-Dinescu et al. (2008) measurement for SA 71 at $l \approx 167^{\circ}$ (Figure 19), and also passes near the spatial location of SA 71 (Figure 20), so it is possible that the debris in these two fields originated from the same progenitor. Ivezić et al. (2008) studied kinematics near the anticenter using thousands of stars from the SDSS database at Galactocentric distances $13 \mathrm{kpc}<R_{\mathrm{GC}}<16 \mathrm{kpc}$, and found an excess of stars with very little vertical (i.e., along Galactic latitude, perpendicular to the disk) motion and rotation velocities faster than the LSR rotation by $\sim 20-50 \mathrm{~km} \mathrm{~s}^{-1}$. At a distance of $15 \mathrm{kpc}$, this results in $-4000 \mathrm{kpc} \mathrm{km} \mathrm{s}^{-1}<L_{Z}<-3500 \mathrm{kpc} \mathrm{km} \mathrm{s}^{-1}$, which is also similar to the angular momenta from both the ACS results and the Mon debris in SA 71. We note, though, that in our result, the GCM08 orbit, and the SA 71 measurement (Casetti-Dinescu et al. 2008), the rotation component of the motion lags the LSR, while Ivezić et al. (2008) find Mon to be faster than the LSR by $\sim 50 \mathrm{~km} \mathrm{~s}^{-1}$. Ultimately, while all of these measurements are finding similar orbital angular momenta, there is still insufficient discriminating power in the results to assess whether the ACS and Mon share a common origin.

It is important to remind the reader that the derived orbit we have shown was integrated in a spherical MW halo. The best fits for Monoceros from Peñarrubia et al. (2005) require an oblate dark matter halo (i.e., $q<1$, where $q$ is the ratio of minor axis to the major axis of the potential) to reproduce the observed characteristics of the detections assumed to be part of the Monoceros system at the time. The difference in halo potentials used may be the source of at least some of the discrepancy between their model and our derived orbits in Figures 19 and 20. Constraints on the halo flattening based on the Sagittarius dSph tidal stream have variously argued that the halo is prolate $(q>1$; Helmi 2004), nearly spherical ( $q=0$; Ibata et al. 2001; Fellhauer et al. 2006), or slightly oblate (Johnston et al. 2005; Law et al. 2005; Martínez-Delgado et al. 2004). More recently, Law et al. (2009) and Law \& Majewski (2010) were able to simultaneously reproduce most observed characteristics of the Sagittarius stream by modeling the halo as triaxial, thus reconciling the prior seemingly conflicting results, wherein a prolate halo was required to match RVs of leading debris, while an oblate halo was needed to get the correct positions along the leading arm. Further constraints on the MW halo shape can be derived by modeling the precession of other tidal streams, and once the ACS and Monoceros systems are well understood, they might be used to trace the low-latitude dark matter structure of the MW. However, for this work we chose simply to use a spherical halo model to assess the general qualitative structure of the orbit implied by the three-dimensional velocity we have measured. A more comprehensive study utilizing all extant data for the ACS and Mon features (which also would require sorting out which data to include for each system) and varying halo parameters is beyond the scope of this work. We simply note here that changing the halo shape (and other parameters, such as the rotation speed at the solar circle or the distance of the Sun from the Galactic center) would alter the exact characteristics of the orbit shown in Figures 13, 19, and 20, but not the general structure of a low-latitude, ring-like feature.

\section{SUMMARY}

We have found that the three-dimensional kinematics of SA 76 stars selected to be ACS members produce unexpected results - the orbit derived from the measured motions does not follow the obvious stream (and the orbit fit to it by GCM08) from the SDSS database, but rather is inclined to it by $\sim 30^{\circ}$. This makes it difficult to reach conclusions about the global structure of the ACS based on our measurements-we are unable to confirm or rule out an association of the ACS with the many Monoceros stream detections or to speculate on a possible progenitor for the system. We do conclude, however, that our findings can best be explained if the ACS debris in SA 76 is part of one of the apparently kinematically cold substreams or tributaries found by Grillmair (2006) to make up the larger ACS stream. In fact, the measured motions of SA 76 ACS debris and the ACS orbit of Grillmair et al. (2008) suggest that the substreams may be the remnants of two (or more) satellites that fell into (and were disrupted by) the MW together. More detailed photometric and chemodynamical studies of the ACS are necessary to explore this possibility. In addition, the association of the EBS with the ACS could possibly be confirmed by studies extending beyond the $\delta \sim 0^{\circ}$ limit of the SDSS footprint.

We thank the anonymous referee for a careful reading of the manuscript, and appreciate useful discussions with Ricardo Muñoz. We gratefully acknowledge support by NSF grant AST-0807945 and NASA/JPL contract 1228235. D.I.C. acknowledges the support of NSF grant AST-0406884. 
Funding for the SDSS and SDSS-II has been provided by the Alfred P. Sloan Foundation, the Participating Institutions, the National Science Foundation, the US Department of Energy, the National Aeronautics and Space Administration, the Japanese Monbukagakusho, the Max Planck Society, and the Higher Education Funding Council for England. The SDSS Web site is http://www.sdss.org/

The SDSS is managed by the Astrophysical Research Consortium for the Participating Institutions. The Participating Institutions are the American Museum of Natural History, Astrophysical Institute Potsdam, University of Basel, University of Cambridge, Case Western Reserve University, University of Chicago, Drexel University, Fermilab, the Institute for Advanced Study, the Japan Participation Group, Johns Hopkins University, the Joint Institute for Nuclear Astrophysics, the Kavli Institute for Particle Astrophysics and Cosmology, the Korean Scientist Group, the Chinese Academy of Sciences (LAMOST), Los Alamos National Laboratory, the Max-PlanckInstitute for Astronomy (MPIA), the Max-Planck-Institute for Astrophysics (MPA), New Mexico State University, Ohio State University, University of Pittsburgh, University of Portsmouth, Princeton University, the United States Naval Observatory, and the University of Washington.

Facilities: WIYN (Hydra), Sloan

\section{REFERENCES}

Abadi, M. G., Navarro, J. F., Steinmetz, M., \& Eke, V. R. 2003, ApJ, 597, 21 Adelman-McCarthy, J. K., et al. 2007, ApJS, 172, 634

Bellazzini, M., Ibata, R., Ferraro, F. R., \& Testa, V. 2003, A\&A, 405, 577

Belokurov, V., et al. 2006, ApJ, 642, L137

Bullock, J. S., \& Johnston, K. V. 2005, ApJ, 635, 931

Casetti-Dinescu, D. I., Carlin, J. L., Girard, T. M., Majewski, S. R., Peñarrubia, J., \& Patterson, R. J. 2008, AJ, 135, 2013

Casetti-Dinescu, D. I., Majewski, S. R., Girard, T. M., Carlin, J. L., van Altena, W. F., Patterson, R. J., \& Law, D. R. 2006, AJ, 132, 2082 (CD06)

Chou, M.-Y., Majewski, S. R., Cunha, K., Smith, V. V., Patterson, R. J., \& Martínez-Delgado, D. 2010, ApJ, 720, L5

Chou, M., et al. 2007, ApJ, 670, 346

Conn, B. C., Lane, R. R., Lewis, G. F., Irwin, M. J., Ibata, R. A., Martin, N. F., Bellazzini, M., \& Tuntsov, A. V. 2008, MNRAS, 390, 1388

Conn, B. C., Lewis, G. F., Irwin, M. J., Ibata, R. A., Ferguson, A. M. N., Tanvir, N., \& Irwin, J. M. 2005, MNRAS, 362, 475

Conn, B. C., et al. 2007, MNRAS, 376, 939

Crane, J. D., Majewski, S. R., Rocha-Pinto, H. J., Frinchaboy, P. M., Skrutskie, M. F., \& Law, D. R. 2003, ApJ, 594, L119

Da Costa, G. S., \& Armandroff, T. E. 1995, AJ, 109, 2533

Dehnen, W., \& Binney, J. J. 1998, MNRAS, 298, 387

Dinescu, D. I., Majewski, S. R., Girard, T. M., \& Cudworth, K. M. 2000, AJ, 120,1892

D’Onghia, E., \& Lake, G. 2009, in IAU Symp. 256, The Magellanic System: Stars, Gas, and Galaxies, ed. J. T. van Loon \& J. M. Oliveira (Cambridge: Cambridge Univ. Press), 473

Dotter, A., Chaboyer, B., Jevremović, D., Kostov, V., Baron, E., \& Ferguson, J. W. 2008, ApJS, 178, 89

Fellhauer, M., et al. 2006, ApJ, 651, 167

Forbes, D. A., Masters, K. L., Minniti, D., \& Barmby, P. 2000, A\&A, 358, 471

Frinchaboy, P. M., Majewski, S. R., Crane, J. D., Reid, I. N., Rocha-Pinto, H. J., Phelps, R. L., Patterson, R. J., \& Muñoz, R. R. 2004, ApJ, 602, L21

Frinchaboy, P. M., Muñoz, R. R., Phelps, R. L., Majewski, S. R., \& Kunkel, W. E. 2006, AJ, 131, 922
Grillmair, C. J. 2006, ApJ, 651, L29 (G06)

Grillmair, C. J. 2009, ApJ, 693, 1118

Grillmair, C. J., Carlin, J. L., \& Majewski, S. R. 2008, ApJ, 689, L117 (GCM08)

Hargreaves, J. C., Gilmore, G., Irwin, M. J., \& Carter, D. 1994, MNRAS, 269, 957

Harris, W. E. 1996, AJ, 112, 1487

Helmi, A. 2004, ApJ, 610, L97

Hernquist, L. 1990, ApJ, 356, 359

Ibata, R. A., Irwin, M. J., Lewis, G. F., Ferguson, A. M. N., \& Tanvir, N. 2003, MNRAS, 340, L21

Ibata, R., Lewis, G. F., Irwin, M., Totten, E., \& Quinn, T. 2001, ApJ, 551, 294

Ivezić, Ž., et al. 2008, ApJ, 684, 287

Jones, L. A. 1998, PhD thesis, Univ. North Carolina at Chapel Hill

Johnson, D. R. H., \& Soderblom, D. R. 1987, AJ, 93, 864

Johnston, K. V., Law, D. R., \& Majewski, S. R. 2005, ApJ, 619, 800

Johnston, K. V., Spergel, D. N., \& Hernquist, L. 1995, ApJ, 451, 598

Kazantzidis, S., Bullock, J. S., Zentner, A. R., Kravtsov, A. V., \& Moustakas, L. A. 2008, ApJ, 688, 254

Kleyna, J., Wilkinson, M. I., Evans, N. W., Gilmore, G., \& Frayn, C. 2002, MNRAS, 330, 792

Law, D. R., Johnston, K. V., \& Majewski, S. R. 2005, ApJ, 619, 807

Law, D. R., \& Majewski, S. R. 2010, ApJ, 714, 229

Law, D. R., Majewski, S. R., \& Johnston, K. V. 2009, ApJ, 703, L67

Li, Y., \& Helmi, A. 2008, MNRAS, 385, 1365

López-Corredoira, M., Momany, Y., Zaggia, S., \& Cabrera-Lavers, A. 2007, A\&A, 472, L47

Luyten, W. J. 1922, Lick Obs. Bull., 10, 135

Majewski, S. R. 1992, ApJS, 78, 87

Majewski, S. R., Munn, J. A., \& Hawley, S. L. 1994, ApJ, 427, L37

Majewski, S. R., et al. 2004, AJ, 128, 245

Martin, N. F., Ibata, R. A., Bellazzini, M., Irwin, M. J., Lewis, G. F., \& Dehnen, W. 2004, MNRAS, 348, 12

Martínez-Delgado, D., Gómez-Flechoso, M. Á., Aparicio, A., \& Carrera, R. 2004, ApJ, 601, 242

Martínez-Delgado, D., et al. 2010, AJ, 140, 962

Miyamoto, M., \& Nagai, R. 1975, PASJ, 27, 533

Moitinho, A., Vázquez, R. A., Carraro, G., Baume, G., Giorgi, E. E., \& Lyra, W. 2006, MNRAS, 368, L77

Momany, Y., Zaggia, S., Gilmore, G., Piotto, G., Carraro, G., Bedin, L. R., \& de Angeli, F. 2006, A\&A, 451, 515

Monaco, L., Bellazzini, M., Bonifacio, P., Buzzoni, A., Ferraro, F. R., Marconi, G., Sbordone, L., \& Zaggia, S. 2007, A\&A, 464, 201

Muñoz, R. R., Carlin, J. L., Frinchaboy, P. M., Nidever, D. L., Majewski, S. R., \& Patterson, R. J. 2006, ApJ, 650, L51

Munn, J. A., et al. 2004, AJ, 127, 3034

Munn, J. A., et al. 2008, AJ, 136, 895

Newberg, H. J., et al. 2002, ApJ, 569, 245

Peñarrubia, J., et al. 2005, ApJ, 626, 128

Pryor, C., \& Meylan, G. 1993, in ASP Conf. Ser. 50, Structure and Dynamics of Globular Clusters, ed. S. G. Djorgovski \& G. Meylan (San Francisco, CA: ASP), 357

Robin, A. C., Reylé, C., Derrière, S., \& Picaud, S. 2003, A\&A, 409, 523

Rocha-Pinto, H. J., Majewski, S. R., Skrutskie, M. F., \& Crane, J. D. 2003, ApJ, 594, L115

Rocha-Pinto, H. J., Majewski, S. R., Skrutskie, M. F., Patterson, R. J., Nakanishi, H., Muñoz, R. R., \& Sofue, Y. 2006, ApJ, 640, L147

Schiavon, R. P. 2007, ApJS, 171, 146

Schlegel, D. J., Finkbeiner, D. P., \& Davis, M. 1998, ApJ, 500, 525

Tonry, J., \& Davis, M. 1979, AJ, 84, 1511

Vivas, A. K., \& Zinn, R. 2006, AJ, 132, 714

Vogt, S. S., Mateo, M., Olszewski, E. W., \& Keane, M. J. 1995, AJ, 109, 151

Wilhelm, R., Beers, T. C., Allende Prieto, C., Newberg, H. J., \& Yanny, B. in ASP Conf. Ser. 336. 2005, Cosmic Abundances as Records of Stellar Evolution and Nucleosynthesis, ed. T. G. Barnes, III \& F. N. Bash (San Francisco, CA: ASP), 371

Yanny, B., et al. 2003, ApJ, 588, 824

Younger, J. D., Besla, G., Cox, T. J., Hernquist, L., Robertson, B., \& Willman, B. 2008, ApJ, 676, L21 